\title{
Mathematical Model of Radiation Effects on Thrombopoiesis in Rhesus Macaques and Humans
}

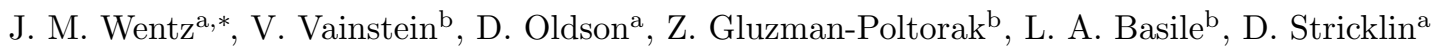 \\ ${ }^{a}$ Applied Research Associates, Inc., Arlington, VA \\ ${ }^{b}$ Neumedicines, Inc., Pasadena, CA
}

\begin{abstract}
A mathematical model that describes the effects of acute radiation exposure on thrombopoiesis in primates and humans is presented. Thrombopoiesis is a complex multistage dynamic process with potential differences between species. Due to known differences in cellular radiosensitivities, nadir times, and cytopenia durations, direct extrapolation from rhesus to human platelet dynamics is unrealistic. Developing mathematical models of thrombopoiesis for both humans and primates allows for the comparison of the system's response across species. Thus, data obtained in primate experiments can be extrapolated to predictions in humans. Parameter values for rhesus macaques and humans were obtained either from direct experimental measurements or through optimization procedures using dynamic data on platelet counts following radiation exposure. Model simulations accurately predict trends observed in platelet dynamics: at low radiation doses platelet counts decline after a time lag, and nadir depth is dose dependent. The models were validated using data that was not used during the parameterization process. In particular, additional experimental data was used for rhesus, and accident and platelet donor data was used for humans. The model aims to simulate the average response in rhesus and humans following irradiation. Variation in platelet dynamics due to individual variability can be modeled using Monte Carlo simulations in which parameter values are sampled from distributions. This model provides insight into the time course of the physiological effects of radiation exposure, information which could be valuable for disaster planning and survivability analysis and help in drug development of radiation medical countermeasures.
\end{abstract}

Keywords: platelet, megakaryocyte, health effects, ordinary differential equation model

\section{Introduction}

Thrombopoiesis is the process of platelet formation. Platelets are responsible for the coagulation of blood and play a role in immunity (Yeaman, 2010). The thrombopoietic system is highly sensitive to ionizing radiation, primarily due to the effect of radiation on hematopoietic stem cells (HSCs) (Kato et al., 2013). Perturbations to platelet counts, including rapid declines and states of thrombocytopenia, are correlated with lethality (Housinger et al., 1993; Akca et al., 2002; Wang et al., 2010). Understanding the dynamics of the thrombopoietic system following radiation exposure can help one predict survivability, understand radiation damage mechanisms, and develop interventions.

Platelet generation is initiated by self-renewing HSCs in the bone marrow. These stem cells differentiate and through a series of mitotic divisions produce megakaryocytes (MKs), the precursors of platelets. As MKs mature, their ploidy increases through a series of endomitoses. Once mature, MKs produce platelets which enter the blood stream. Mathematical models have been

\footnotetext{
* Corresponding author

Email address: jwentz@ara.com (J. M. Wentz)
}

developed to describe how these processes are affected by radiation exposure (Smirnova, 2010, 2012; Graessle, 2000), chemotherapy treatment (Scholz et al., 2010), pegylated recombinant MK growth and development factor (Harker et al., 2000), and thrombopoietin (TPO) (Skomorovski et al., 2003). In addition, we have adapted O. A. Smirnova's radiation injury model to study how burn injury affects thrombopoiesis (Wentz et al., 2014a).

In this study, we use Smirnova's radiation exposure model as a starting point (Smirnova, 2010, 2012). Smirnova's models provide reasonable predictions of the hematopoietic response in humans after acute radiation exposure. However, this work seeks to (1) adapt Smirnova's model to accurately predict thrombopoiesis in rhesus and (2) parameterize the model by incorporating as many welldocumented experimentally determined parameters as possible. In order to better correlate certain trends observed with experimental data, we made modifications to the model structure. This new model structure was re-parameterized to predict the platelet response in rhesus and humans following a wide range radiation dose levels. One significant structural change between Smirnova's model and the model here is the addition of transit subcompartments to simulate time delays and introduce an age-structure into the model. Additionally, the model incorporates different 
Table 1: State variables in the human and rhesus thrombopoiesis models.

\begin{tabular}{ll}
\hline State Variable & Biological Representation \\
\hline$x_{1}^{u d}$ & Undamaged cells in the mitotic compart- \\
& ment $X_{1}$ \\
$x_{2 I, i}^{u d}$ & Undamaged immature MKs in the $i^{\text {th }}$ \\
& subcompartment of $X_{2 I}$ \\
$x_{2 M, i}^{u d}$ & Undamaged mature MKs in the $i^{\text {th }}$ sub- \\
& compartment of $X_{2 M}$ \\
$x_{3, i}$ & Undamaged circulating platelets in the \\
$x_{1}^{d}$ & $i^{\text {th }}$ subcompartment of $X_{3}$ \\
& Damaged cells in the mitotic compart- \\
$x_{2}^{d}$ & ment $X_{1}$ \\
\hline
\end{tabular}

feedback mechanisms, MK radiosensitivity, and alternate models of radiation damage (see Model Development).

The development of a rhesus model followed by a human model adds model validity for several reasons. First, rhesus data comes from a controlled environment, which eliminates confounding factors such as extensive supportive care treatment and blood transfusions. This also helps one identify structures to include in a model. Observed changes in platelet count are driven only by the insult and physiological response. Developing a rhesus model alongside a human model is also the first step in translating effects from rhesus to humans.

In the sections that follow, we describe a mechanistic model of the effect of acute radiation exposure on thrombopoiesis in rhesus and humans. Extensive supportive care is excluded from this model. Using this model one can simulate, up to 30 days following exposure, radiation-induced perturbations in peripheral platelet dynamics and progenitor cell dynamics.

\section{Methods}

\subsection{Model Development}

We adopt a simplified view of the thrombopoietic system, as illustrated in Figure 1, by grouping cells into compartments based on degree of maturity, differentiation, and location in the body (e.g. bone marrow, circulation). Similar to Smirnova (2010), these compartments include the mitotic bone marrow precursor cells (HSCs to megakaryoblasts, $\left.X_{1}\right)$, MKs in the bone marrow $\left(X_{2}\right)$, and platelets in circulation $\left(X_{3}\right)$ (see Table 1 for a complete list of state variables and their biological interpretation). Normal transitions among the compartments are indicated by solid arrows at the top of Figure 1, and dotted lines represent homeostatic feedback mechanisms. The effect of radiation on the thrombopoietic system is modeled by the transition of radiosensitive cells into additional compartments that essentially sequester the damaged cells: there is no further maturation or differentiation of these cells, though they continue to modulate homeostatic feedback until they leave the model system through early death.
For the complete list of model equations, see Appendix A.1.

This conceptual model of thrombopoiesis was applied to both non-human primates (NHPs) and humans. NHP data provided key insight into model structure because of the precise experimental conditions that could be obtained in these studies.

\subsubsection{Mitotic progenitor compartment}

The mitotic progenitor compartment contains early thrombopoietic progenitors in the bone marrow ranging from self-replicating HSCs through megakaryoblasts. Cells in this compartment are capable of dividing and can also become further differentiated, thereby leaving the compartment. The rate of change of concentration of undamaged mitotic progenitors, $x_{1}^{u d}$, is given by (Smirnova, 2010)

$$
\frac{d x_{1}^{u d}}{d t}=B x_{1}^{u d}-\gamma x_{1}^{u d}
$$

where $\gamma$ is the constant rate of transition into the $X_{2}$ compartment and $B$ is the time-dependent rate of mitotic progenitor repopulation.

To incorporate homeostatic feedback into the repopulation rate $B$, we assume that $B$ is positively correlated with the amount of a generic stimulatory mediator within the system. In particular, we assume that

$$
B=\frac{\alpha}{1+\frac{K}{I}}
$$

where $\alpha$ is the maximum repopulation rate, $K$ is the stimulation constant, and $I$ is the concentration of the generic mediator. Equation 6 is similar to the HSC repopulation rate defined by Smirnova (2010); however, in Smirnova's model the repopulation rate $B$ is negatively correlated with the mediator concentration $I$ (i.e., the mediator is inhibitory). We redefined the mediator in the system such that $I$ is stimulatory because this is consistent with the effect of the principle mediator of thrombopoiesis, TPO (Kaushansky, 2005). Ultimately, the mediator $I$ is intended to represent the combined effect of all biological factors, both inhibitory and stimulatory, that modulate the repopulation rate. These factors include spatial constraints within the bone marrow environment and cytokines.

To close the feedback loop we now need to relate $I$ to the number of cells in our model of the thrombopoiesis system. We therefore assume that all cells in the system, including mitotic progenitors, MKs, and platelets, contribute to the degradation of $I$. For example, the degradation of $I$ due to an overabundance of $X_{1}$ cells represents negative feedback due to bone marrow spatial constraints, and the degradation of $I$ due to $X_{2}$ and $X_{3}$ cells represents the removal of TPO via receptor interactions. This feedback loop is analogous to that developed by Smirnova (2010) where all cells lead to the generation of the inhibitory mediator. 


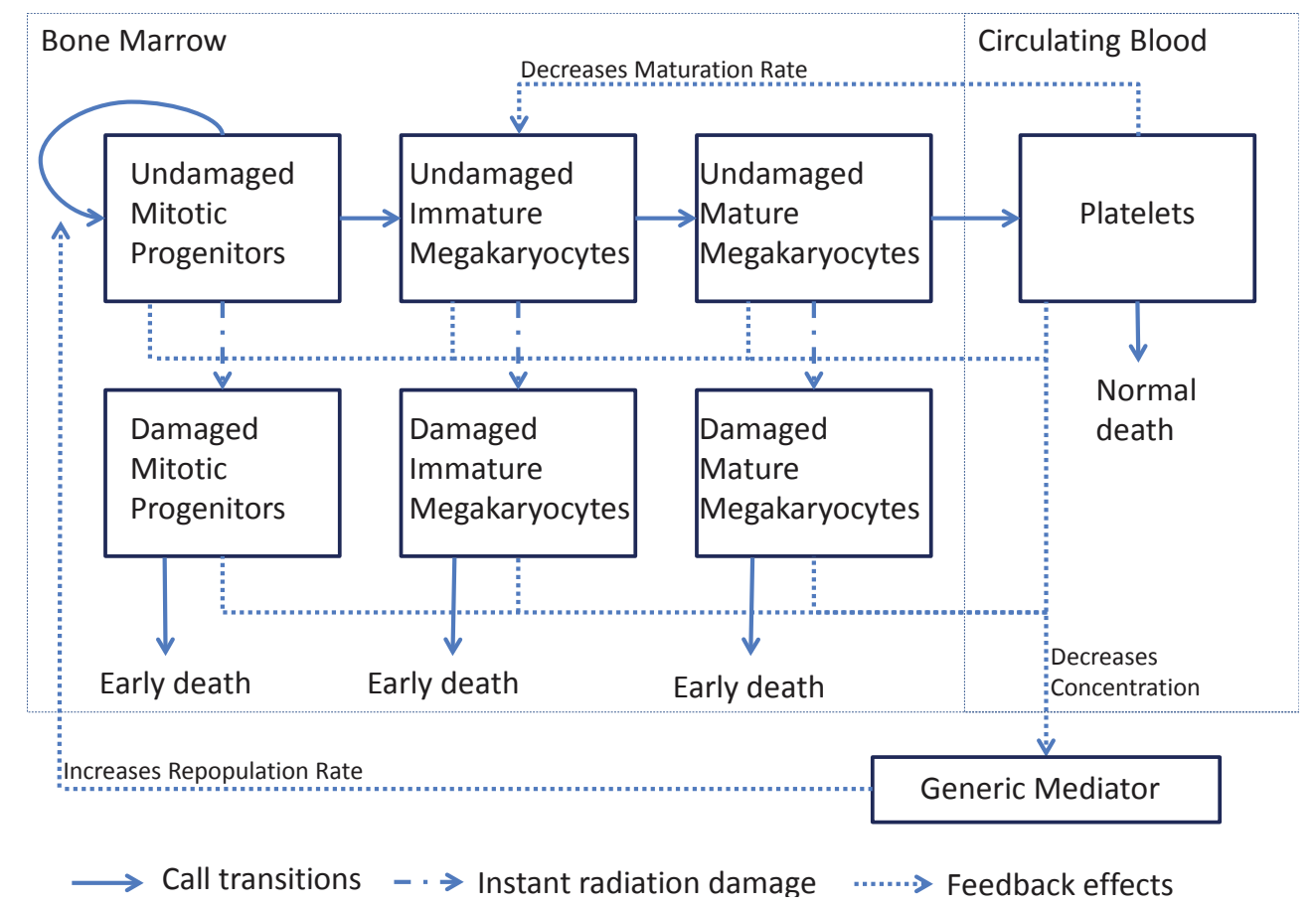

Figure 1: Model of effects of radiation on thrombopoiesis in rhesus and humans.

Assuming that $I$ is generated at a constant rate, the concentration of $I$ changes according to

$$
\frac{d I}{d t}=G-H\left(x_{1}^{u d}+x_{1}^{d}+\theta_{2}\left(x_{2}^{u d}+x_{2}^{d}\right)+\theta_{3} x_{3}\right) I
$$

where $\theta_{2}$ and $\theta_{3}$ represent the relative rate of MK- and platelet-mediated $I$ degradation, respectively (relative to the effect of mitotic progenitors).

The parameters $\theta_{i}$ thus quantify the relative impact of $X_{i}$ cells on the repopulation rate feedback signal. We calculate $\theta_{2}$ through optimization and use experimental data to map $\theta_{2}$ to $\theta_{3}$. To construct this mapping, we first note that platelets and MKs regulate TPO concentration through receptor interactions. Specifically, receptors on the membranes of platelets and MKs bind to and engulf TPO, removing it from the system. We therefore use a receptor count ratio to estimate the amount of TPO removed by platelets relative to the amount removed by MKs. In humans, the average TPO receptor count on peripheral and cord blood MKs is approximately 934 (Kuwaki et al., 1998). In contrast, the TPO receptor count on platelets is approximately $30 \pm 4$ (Broudy et al., 1997). Thus, each MK destroys 934/30 times as much TPO as each platelet, so we assume

$$
\theta_{3}=0.03 \cdot \theta_{2}
$$

In summary, to map $\theta_{2}$ to $\theta_{3}$, we have assumed that (i) platelets and MKs modulate the repopulation rate only through receptor-mediated destruction of TPO, and (ii) the relative amount of TPO degradation due to platelets and MKs depends only on their TPO receptor count ratio. Finally, although Equation 4 was determined using data in humans, it was assumed to hold for rhesus as well.

Using the same approach as Smirnova (2010), we reduce the feedback model given by Equation 2-3 by assuming that I's characteristic time scale is much shorter than the time scales over which the cell populations change. Using a quasi-steady-state approximation for $I$, we assume $d I / d t=0$ and then solve Equation 3 for $I$ :

$$
I=\frac{G}{H\left(x_{1}^{u d}+x_{1}^{d}+\theta_{2}\left(x_{2}^{u d}+x_{2}^{d}\right)+\theta_{3} x_{3}\right)}
$$

Substituting this expression for $I$ into Equation 2 yields

$$
B=\frac{\alpha}{1+\beta\left(x_{1}^{u d}+x_{1}^{d}+\theta_{2}\left(x_{2}^{u d}+x_{2}^{d}\right)+\theta_{3} x_{3}\right)}
$$

where $\beta=H K / G$.

$\beta$ can be determined as a function of kinetic rates and the healthy, steady-state values of $x_{1}^{u d}, x_{2}^{u d}$, and $x_{3}$ $\left(\bar{x}_{1}^{u d}, \bar{x}_{2}^{u d}\right.$, and $\bar{x}_{3}$, respectively). Under steady-state conditions, the concentration of mitotic progenitors is constant $\left(d x_{1}^{u d} / d t=0\right)$ and there are no damaged cells. Thus, $B=\gamma$ (see Equation 1) and, $x_{i}^{d}=0$ for $i=1,2$. Making these substitutions into Equation 6 yields

$$
\gamma=\frac{\alpha}{1+\beta\left(\bar{x}_{1}^{u d}+\theta_{2} \bar{x}_{2}^{u d}+\theta_{3} \bar{x}_{3}\right)}
$$

Rearranging, we see that

$$
\beta=\frac{\alpha / \gamma-1}{\bar{x}_{1}^{u d}+\theta_{2} \bar{x}_{2}^{u d}+\theta_{3} \bar{x}_{3}}
$$


Equation 2 implies that the maximum rate of mitotic progenitor repopulation is equal to $\alpha$. In Smirnova's model. $\alpha$ is assumed to equal $2.0 d^{-1}$ in humans (Smirnova, 2010). Using an exponential growth model, this implies that the time needed for cellular division is

$$
T_{d i v}=\frac{\ln (2)}{2 d^{-1}} \cdot \frac{24 h}{1 d}=8.3 h
$$

which is consistent with the time required for DNA synthesis (Andreeff et al., 2000). Thus, $2.0 d^{-1}$ is a biologically reasonable maximum rate of maturation, and we use it in both the human and rhesus models presented here.

Here, the cellular division time is assumed to equal the DNA synthesis time as cell concentrations approach zero. A more accurate method would be to optimize $\alpha$, using $2.0 d^{-1}$ as an upper bound; however, because the parameters $\alpha$ and $\gamma$ were found to be highly correlated in the optimization, a value for one of these parameters had to be set.

\subsubsection{MK compartment}

After MKs are produced through stem cell differentiation and mitotic division, the MKs enter a maturation phase characterized by a gradual increase in both mass and ploidy level (Kaushansky, 2005). It is only after MKs have matured that they produce platelets that enter the blood. A time delay, therefore, exists between events that selectively damage HSCs and downstream effects such as perturbations in platelet count. For example, in both rhesus and humans, there is a delay between radiation exposure and decline in the peripheral platelet count (Authier et al., 2013; Bond et al., 1965).

To introduce a maturity structure into the model and represent the MK maturation delay, we divide the MK compartment, $X_{2}$, into $n$ subcompartments. Cells in the first $n / 2$ subcompartments, $X_{2 I, i}$, are called immature MKs, and cells in the second $n / 2$ subcompartments, $X_{2 M, i}$, are called mature MKs. Moreover, as described below, the transition rates among the first $n / 2$ subcompartments depend on platelet concentration, so the model essentially describes a state-dependent MK maturation delay.

The model equations for the MK compartment are

$$
\begin{aligned}
& \frac{d x_{2 I, 1}^{u d}}{d t}=\gamma x_{1}^{u d}-n \delta x_{2 I, 1}^{u d} \\
& \frac{d x_{2 I, i}^{u d}}{d t}=n \delta x_{2 I, i-1}^{u d}-n \delta x_{2 I, i}^{u d} \quad(i=2,3, \ldots, n / 2) \\
& \frac{d x_{2 M, 1}^{u d}}{d t}=n \delta x_{2 I, n / 2}^{u d}-n \delta_{0} x_{2 M, 1}^{u d} \\
& \frac{d x_{2 M, i}^{u d}}{d t}=n \delta_{0} x_{2 M, i-1}^{u d}-n \delta_{0} x_{2 M, i}^{u d} \quad(i=2,3, \ldots, n / 2)
\end{aligned}
$$

where $x_{2 I, i}$ and $x_{2 M, i}$ for $i=1,2, \ldots, n / 2$ are state variables that represent subcompartment $i$ in the immature and mature MK compartments, respectively. $\gamma x_{1}^{u d}$ represents the

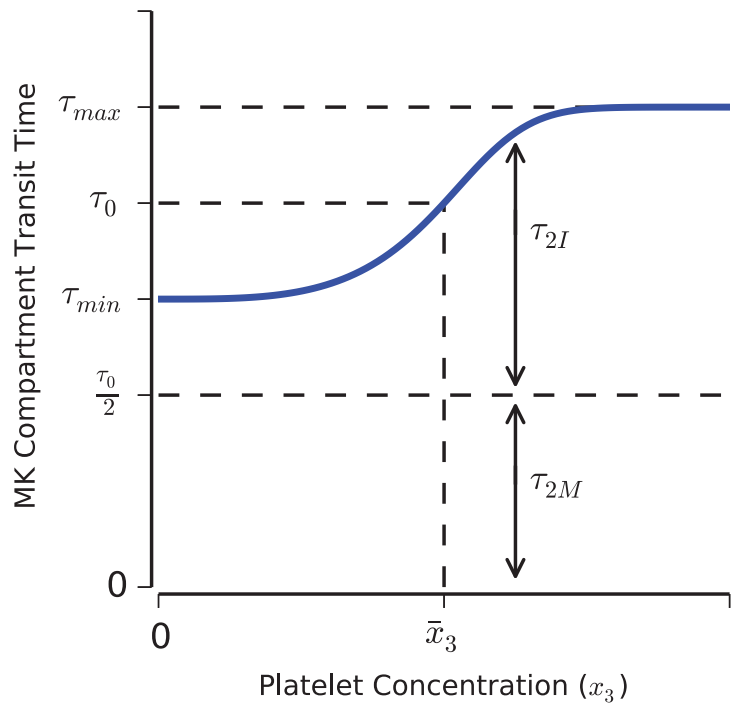

Figure 2: Feedback effect of platelets on MK transit time. As the platelet blood concentration $x_{3}$ increases, the immature MK transit time $\left(\tau_{2 I}\right)$ increases while the mature MK transit time $\left(\tau_{2 M}\right)$ remains constant. $\tau_{\min }$ represents the minimum MK transit time, $\tau_{\text {max }}$ represents the maximum MK transit time, and $\tau_{0}$ is the transit time when $x_{3}=\bar{x}_{3} .\left(\tau_{2 I}=1 / 2 \delta, \tau_{2 M}=1 / 2 \delta_{0}.\right)$

influx from the $X_{1}$ compartment, $\delta_{0}^{-1}$ is the expected MK transit time under healthy, steady-state conditions, and $\delta$ is a variable rate that determines how the transit time through the immature MK subcompartments changes in response to perturbations in the platelet concentration.

We have thus introduced a second feedback mechanism, represented by the rate $\delta$, whereby the platelet concentration $x_{3}$ modulates maturation rate in the $X_{2 I, i}$ subcompartments (see Figure 1). In Smirnova's 2010 model the rate of MK maturation is not variable and there is instead an amplification factor which is dependent on platelet concentration $x_{3}$. The revised feedback mechanism used here is based on a review of experimental data analyzing TPO and platelet dynamics. In particular, TPO is known to affect MK maturation; however, TPO does not affect the formation of platelets from the MK cytoplasm (Kaushansky, 2005). Following thrombopheresis in humans, platelet counts return to normal levels at the expected rate for the first 3-4 days given known production rates (Sullivan et al., 1977). However, after the fourth day following thrombopheresis, there is a subsequent increase in the platelet production rate, suggesting a negative feedback effect of platelet concentration on the maturation rate of early MKs. It is for these reasons that we assume platelet concentration affects the immature MK maturation rate, but not the transit time through the $X_{2 M}$ compartment (see Figure 2).

We assume this effect depends only on the platelet concentration. However, the mechanism may involve TPO which can be degraded by MKs. Although, the MK con- 
centration could ultimately affect transit time through the $X_{2 I}$ compartment, this potential mechanism is not included in our model. This was due to the lack of available data on the contribution of MKs to this mechanism.

To complete the description of the second feedback loop we must now define how the rate $\delta$ depends on the platelet concentration $x_{3}$. In other words, we need to define how the immature MK transit time $\tau_{2 I}$ depends on $x_{3}$. First, the MK compartment total transit time function $\left(\tau_{2 M}+\tau_{2 I}\right)\left(x_{3}\right)$ should have the following attributes (see Figure 2): (1) $\left(\tau_{2 M}+\tau_{2 I}\right)\left(\bar{x}_{3}\right)=\tau_{0}$, i.e., at steadystate, the transit time through all $n$ MK subcompartments should be $\tau_{0},(2)$ as the platelet concentration approaches zero, $\left(\tau_{2 M}+\tau_{2 I}\right)$ should approach a minimum value $\tau_{\text {min }}$, and (3) as the platelet concentration approaches infinity, $\left(\tau_{2 M}+\tau_{2 I}\right)$ should approach a maximum value $\tau_{\max }$. The values of $\tau_{0}=1 / \delta_{0}, \tau_{\min }$, and $\tau_{\max }$ correspond to experimental measurements of steady-state, minimum, and maximum MK maturation times (respectively).

Next, we allocate a fixed portion of the MK total transit time to the $X_{2 M}$ compartment: $\tau_{2 M}\left(x_{3}\right) \equiv \tau_{0} / 2$ (and therefore we assume $\tau_{\min }>\tau_{0} / 2$; see Figure 2). Given this fixed value of $\tau_{2 M}$, the above three criteria for the function $\left(\tau_{2 M}+\tau_{2 I}\right)\left(x_{3}\right)$ imply the following three criteria for the function $\tau_{2 I}\left(x_{3}\right)$ : $\left(1^{\prime}\right) \tau_{2 I}\left(\bar{x}_{3}\right)=\tau_{0} / 2$, i.e., at steadystate, the transit time through the $n / 2 X_{2 I, i}$ subcompartments should be $\tau_{0} / 2,\left(2^{\prime}\right)$ as the platelet concentration approaches zero, $\tau_{2 I}$ should approach a minimum value $\tau_{2 I}^{\text {min }}=\tau_{\text {min }}-\tau_{0} / 2$, and $\left(3^{\prime}\right)$ as the platelet concentration approaches infinity, $\tau_{2 I}$ should approach a maximum value $\tau_{2 I}^{\max }=\tau_{\max }-\tau_{0} / 2$. To model a sigmoidal response that satisfies $\left(1^{\prime}\right),\left(2^{\prime}\right)$, and $\left(3^{\prime}\right)$, we define

$\tau_{2 I}\left(x_{3}\right)=\left(\frac{1}{\tau_{2 I}^{\max }}+\left(\frac{1}{\tau_{2 I}^{\min }}-\frac{1}{\tau_{2 I}^{\max }}\right)\left(\frac{\frac{1}{\tau_{0} / 2}-\frac{1}{\tau_{2 I}^{\max }}}{\frac{1}{\tau_{2 I}^{\min }}-\frac{1}{\tau_{2 I}^{\max }}}\right)^{\left(\frac{x_{3}}{\bar{x}_{3}}\right)^{\lambda}}\right)$

where $\lambda$ determines the feedback strength and $\bar{x}_{3}$ is the concentration of platelets under healthy, steady-state conditions. In Figure 2 the MK compartment transit time is plotted using hypothetical values for $\tau_{0}, \tau_{\min }, \tau_{\max }$, and $\lambda$. This feedback function was adapted from Scholz et al. (2010), in which a similar function was used to describe how TPO increases the maturation rate of progenitor cells. Finally, returning to defining how the rate $\delta$ depends on the platelet concentration $x_{3}$, there are $n / 2 X_{2 I, i}$ subcompartments, so we set $1 / n \delta$ equal to $\tau_{2 I} /(n / 2)$ (see Equations 10-11). In other words, we define

$$
\delta\left(x_{3}\right)=\frac{1}{2 \tau_{2 I}\left(x_{3}\right)}
$$

Values for the parameters $\tau_{\max }$ and $\tau_{\min }$ were determined by assuming $\tau_{0} / 4 \leq \tau_{2 I} \leq 3 \tau_{0} / 4$. Under this assumption, $\tau_{\max }=5 \tau_{0} / 4$ and $\tau_{\min }=3 \tau_{0} / 4$. Though there is little experimental data that supports our assumption regarding $\tau_{2 I}$, we note that it has been observed that when
$92 \%$ of platelets were removed from the circulation of rats, the MK maturation time decreased to $62-85 \%$ of control values (Ebbe et al., 1968). This provides some validation for setting the minimum MK transit time equal to $75 \%$ of normal. However, the same study notes that sustained transfusion-induced thrombocytosis did not result in a suppressed MK maturation rate in rats. Thus, verifying whether thrombocytosis reduces MK maturation rate requires future experimental work.

\subsubsection{Platelet compartment}

The platelet compartment consists of all platelets in the blood stream. The platelet compartment is divided into $m$ subcompartments to give it a maturity structure and to generate more biologically realistic transit time distributions (Murphy and Francis, 1971). This is an alteration to Smirnova's 2010 model in which there is only one platelet compartment. Fitting human platelet data to gamma functions suggests a value for $m$ of 7 or 11 (Dowling et al., 2010; Scholz et al., 2010). Here, 9, the midpoint of that range, was chosen for $m$ in the human model, and it was assumed to be the same in the rhesus model. The concentration of platelets in each subcompartment changes with time according to

$$
\begin{aligned}
& \frac{d x_{3,1}}{d t}=\sigma n \delta_{0} x_{2 M, n / 2}^{u d}-m \psi x_{3,1} \\
& \frac{d x_{3, i}}{d t}=m \psi x_{3, i-1}-m \psi x_{3, i} \quad(i=2,3, \ldots, m)
\end{aligned}
$$

where $x_{3, i}$ represents the concentration of platelets in the platelet subcompartment $i . n \delta_{0} x_{2 M, n / 2}^{u d}$ is the efflux from the MK compartment and $\sigma$ is an amplification factor. $\bar{\sigma}^{1}$ represents the number of platelets produced per MK, which is approximately 3000 in humans and assumed to be the same in rhesus (Deutsch and Tomer, 2013). In humans, the lifespan of a platelet is approximately 9 days (Schmitt et al., 2001; Mezzano et al., 1982; Kaushansky, 2005; Finch et al., 1977). Thus, in the human model the value of $\psi$ was set to $1 / 9$ or $0.11 \mathrm{~d}^{-1}$. In rhesus, fitting platelet decay to a gamma distribution led to an expected platelet lifespan of $5.5 \mathrm{~d}$ (Harker et al., 1997), leading to $\psi=0.18 d^{-1}$.

\subsubsection{Radiation damage}

Following radiation exposure, we assume cells instantaneously transition into the damaged compartments. In a real-life scenario, acute radiation exposure would occur over a certain time interval of minutes/hours causing damage during this entire interval. However, because the model is intended to capture a lower resolution of response (i.e., days), we can assume that acute exposure is instantaneous. Thus, the proportion of cells damaged is purely dependent on the total dose received and not the dose rate. The input dose to the model is intended to be a gamma free-in-air dose. Once cells become damaged they stop the maturation process and decay at a fixed rate. In 
our model, mitotic progenitors and MKs are radiosensitive, while platelets are assumed to be radioresistant (Greenberg et al., 1968). This is in contrast to Smirnova's 2010 thrombopoiesis model, in which MKs are radioresistant.

Damaged cells are assumed to die during interphase or the next mitosis through either necrotic of apoptotic pathways. In Smirnova's thrombopoiesis model there are two damaged compartments from which cells decay at different rates (Smirnova, 2010). However, we have simplified this model by using only one damaged compartment. Based on the platelet dynamic data collected, including more than one compartment did not significantly improve optimizations. The rate of decay $\mu$ from the damaged compartment was determined using the rates of decay from the two damaged compartments in Smirnova's original model which she obtained from the literature. Specifically the reciprocal of these rates $(0.16 \mathrm{~d}$ and $2.0 \mathrm{~d})$ where added together and divided by 2 to find the average of the expected lifespan. $\mu$ was then set as the reciprocal of this value $\left(\mu=1.0 d^{-1}\right)$.

To model cell survivability following radiation exposure, we use the single-target, multi-hit model of radiation damage (Joiner, 2009). In this model, cells have multiple targets which must all be hit by radiation for a cell to become damaged. The fraction of surviving cells $S$ following acute radiation dose $D$ is

$$
S_{i}=1-\left(1-e^{-D / D_{i}^{0}}\right)^{n_{i}}
$$

where $i=1,2\left(S_{1}\right.$ is the undamaged proportion of mitotic progenitors and $S_{2}$ is the undamaged proportion of MKs). $D_{i}^{0}$ is the dose at which there is a $37 \%$ probability that a specific target is not hit and $n_{i}$ is the number of targets per cell. Thus, as $D_{i}^{0}$ increases the cell becomes less radiosensitive, and as $n_{i}$ increases there is a larger shoulder in the dose response curve, meaning at lower doses each cell's likelihood of becoming damaged is small.

Data from in vitro experiments on MK colony forming units (CFU-MKs) following radiation exposure was used to estimate mitotic cell damage in vivo. Here we assume that CFU-MK radiosensitivity represents the radiosensitivity of all mitotic progenitors. In humans cells, the $D_{1}^{0}$ and $n_{1}$ values based on CFU-MK experiments when stimulated with only TPO were 0.79 Gy and 1, respectively (Kashiwakura et al., 2000). In rhesus, due to a lack of experimental data we assume $n_{1}$ is equal to the value in humans. Using a study on cynomolgus monkeys, $D_{1}^{0}$ was determined to equal $1.18 \mathrm{~Gy}$ in the rhesus model (Hrodin et al., 2007) (it was assumed that cynomolgus and rhesus monkey cells have similar radiosensitivities). Besides in vitro verse in vivo, one source of error with these assumptions is that more attenuation of the radiation is likely to occur in in vivo situations before the radiation hits the mitotic progenitors in the bone marrow.

In humans, at low radiation doses there is a significant delay in platelet decline relative to the exposure, which does not happen at doses greater than approximately 5 Gy (Hempelmann et al., 1952; Andrews et al., 1961; Bond et al., 1965; Jammet et al., 1959; Yarmonenko, 1988; Baranov et al., 1994; Miller et al., 1958). This suggests MKs are becoming damaged only at high radiation doses which is in agreement with other studies showing MKs are more radioresistant than other bone marrow cells (Heylmann et al., 2014). Thus, we set $n_{2}$ to a value greater than 1 to simulate the lack of damage at low radiation doses while $D_{2}^{0}$ was optimized. Both $n_{2}$ and $D_{2}^{0}$ were not simultaneously included in the optimization because there was a high collinearity between these two parameters. Multiple combinations of $n_{2}$ and $D_{2}^{0}$ resulted in the same model likelihood. The number of hits required to kill a MK was set to $4\left(n_{2}=4\right)$ in both the human and rhesus models after incrementally testing integers less than 4 . It was determined this was adequate for capturing the early dose response.

\subsection{Data Collection}

Data for optimization and subsequent validation of the model were obtained from experimental animal studies and human case studies. Data for the rhesus optimizations was obtained from well-controlled studies performed in rhesus macaques exposed to various levels of radiation (Basile et al., 2012; Gluzman-Poltorak et al., 2014a,b; Authier et al., 2013; Neumedicines, Inc.). Data for the human optimizations was obtained from Hempelmann et al. (1952); Andrews et al. (1961); Bond et al. (1965); Jammet et al. (1959); Yarmonenko (1988); Baranov et al. (1994); Miller et al. (1958). Tables B4 and B5 provide a summary of the radiation data used in optimizing the rhesus and human model, respectively. Information on the reference, radiation source, dose, and baseline control values is given for all optimization data. For human case studies, dose information was often presented in the literature as a range. In these cases, the midpoint of the dose range was used for optimization and subsequent comparison.

All the data were normalized by baseline control values. If multiple pre-radiation baseline values were obtained, the average of the baseline values was used. Each individual's observational dataset was normalized by the baseline value specific to that individual prior to optimization. In some cases, pre-radiation values for human subjects were not available. When this occurred, the mean of up to four data points collected within 3 days of radiation exposure was used as the baseline. The assumption with this calculation is that the platelet values do not change significantly within the first three days of radiation exposure. Of the 12 subjects whose baseline values were calculated using this method, 9 values are within the normal range observed in humans of $140-440 \cdot 10^{3} \mu L^{-1}$ (Valentin, 2002), one of the values is larger than this range, and two of the values are lower. If no data was collected within three days of exposure, the baseline value was set equal to the mean platelet concentration observed in humans $\left(250 \cdot 10^{3} \mu L-1\right.$; Valentin (2002)).

Data from 254 rhesus macaques with doses ranging from 4 to 12.1 Gy were included in the optimization of the 
rhesus model while 17 human case studies with doses ranging from 0.12 to 12.5 Gy were included in the optimization of the human thrombopoiesis model. Human optimization data were carefully selected in order to represent a range of doses $(0.12-12.5 \mathrm{~Gy})$ and minimize the uncertainty and confounding factors affecting the data. Thus, studies included in the optimization had the most accurate dose estimates, minimal treatment, and relatively uniform and acute gamma exposures. Radiotherapy studies involving cancer patients where the cancer was not related to hematopoiesis were also given priority because many subjects from these studies had baseline platelet count recordings.

\subsection{Parameter Optimization and Confidence Interval Cal- culations}

To optimize parameters, quantify parameter uncertainty, and assess model identifiability we used nonlinear least squares curve fitting and Markov Chain Monte Carlo sampling. All ordinary differential equation (ODE) simulations, optimization procedures, and parameter confidence interval calculations were performed using $\mathrm{R}$ v3.0.0 ( $\mathrm{R}$ Core Team, 2013).

First, to ensure equal weighting of residuals from different data sets, data values were normalized by scaling each value by baseline control values. An optimized parameter set $p_{L M}$ was then obtained using the LevenbergMarquardt algorithm (Mor, 1978) implemented in the modCost and modFit tools from the FME package (Soetaert and Petzoldt, 2010). As part of this process, solution curves for Equations 1, 10-13, 16-17 were calculated using the ode function from the deSolve package (Soetaert et al., 2010).

Next, using the parameter set $p_{L M}$ to initialize a Markov Chain, the Markov Chain Monte Carlo (MCMC) method with the adaptive Metropolis algorithm was used (Laine, 2008; Haario et al., 2001) to estimate 95\% confidence intervals for parameters. This algorithm was performed in $\mathrm{R}$ using modMCMC, a function of the FME library. Finally, to determine whether the models were identifiable the collinearity value was determined using the collin function from the FME package in R (Brun et al., 2001).

In the material that follows, ranges given for optimized parameters represent $95 \%$ confidence interval obtained from this MCMC analysis.

\section{Results and Discussion}

\subsection{Mathematical model of thrombopoiesis reproduces ob- served trends in platelet dynamics following acute ra- diation exposure in rhesus and humans}

The rhesus and human thrombopoiesis models were optimized using platelet dynamic data from rhesus and humans following radiation exposure. The finalized parameter values for the model are shown in Table 2. Both the rhesus and human thrombopoiesis models accurately capture dose-dependent trends observed following acute radiation exposure. Figure 3 shows the thrombopoiesis model simulations at increasing radiation dose levels for both rhesus and humans and compares simulations to select experimental and observational optimization data. All other comparisons between model simulations and optimization data are given in Figure B1 for the rhesus model and in a publicly available technical report for the human model (Wentz et al., 2014b). The purpose of these comparisons is to verify that trends observed in the data are reflected by the models. To further assess the fit of the model, a plot of the model predictions compared with the observed data used in the optimization is given for both rhesus and humans (Figure 4). Figure 3 shows that at low acute radiation doses, there is a plateau phase prior to a decrease in platelet count. The duration of this phase represents the time needed for MK maturation. In rhesus this plateau phase is shorter suggesting a faster MK maturation time in rhesus compared with humans. This is also reflected in the optimized value for $\delta_{0}$ which leads to an expected MK maturation time of 3.8 (3.7-4.0) days in rhesus and 16.7 (14-20) days in humans. This predicted value for the human MK maturation time is somewhat larger than the values given by other studies and mathematical models which range from 4 to 10 days (Smirnova, 2010; Bond et al., 1965; Scholz et al., 2010); however, a larger expected MK maturation time was necessary to capture the duration of the plateau in platelet counts following radiation exposure which in some studies lasted more than 20 days. Therefore, it is possible that in our model we effectively include in the MK compartment some MK progenitors which show similar radiosensitivity patterns as MKs (i.e. some precursors are also not damaged at low radiation doses). Smirnova used the notion of an abortive rise rather than an MK maturation delay to simulate the plateau (Smirnova, 2010). In this phenomenon, a proportion of damaged cells reproduce and behave normally for a certain time period prior to apoptosis (Bond et al., 1965). However, in this version of the model an immediate decrease in platelet counts is still observed at low doses which is not supported by experimental data. Thus, we decided that adding a MK maturation delay was more supported by biological data. In reality, both these processes may be interplaying and causing the observed response.

As the radiation dose increases, the plateau phase diminishes in the human model but remains in the rhesus model. This behavior is in agreement with platelet dynamic data. The radiation threshold for induction of MK damage is lower in humans than in rhesus, leading to the observed differences between the model predictions The increased sensitivity is reflected by the optimized value of $D_{2}^{0}$ which is $2.77(2.59-2.93)$ Gy in the human model and approaches the upper bound of 50 Gy in the rhesus model, implying that rhesus MKs are radioresistant. MK radiosensitivity was engineered into the model as the hypothesized cause for the elimination of the plateau phase at higher doses. The inclusion of MK radiosensitivity is 
Table 2: Parameter values for thrombopoiesis model

\begin{tabular}{|c|c|c|c|c|c|}
\hline \multirow[t]{2}{*}{ Parameter } & \multirow[t]{2}{*}{ Description } & \multicolumn{2}{|c|}{ Rhesus } & \multicolumn{2}{|r|}{ Human } \\
\hline & & Value & Source & Value & Source \\
\hline$\alpha$ & $\begin{array}{l}\text { Maximum rate of mitotic progenitor cell } \\
\text { repopulation }\end{array}$ & $2.0 d^{-1}$ & $\rightarrow$ & $2.0 d^{-1}$ & Assumed \\
\hline$\gamma$ & Rate of mitotic progenitor cell maturation & $0.06 d^{-1}$ & Optimized & $0.26 d^{-1}$ & Optimized \\
\hline$\delta_{0}$ & $\begin{array}{l}\text { Rate of MK maturation when system is at } \\
\text { equilibrium }\end{array}$ & $0.26 d^{-1}$ & Optimized & $0.06 d^{-1}$ & Optimized \\
\hline$\psi$ & Rate of platelet decay & $0.18 d^{-1}$ & $\begin{array}{l}\text { Harker et al. } \\
(1997)\end{array}$ & $0.11 d^{-1}$ & $\begin{array}{l}\text { Mezzano et al. (1982); } \\
\text { Finch et al. (1977) }\end{array}$ \\
\hline$n$ & Number of MK subcompartments & 10 & Assumed & 10 & Assumed \\
\hline$m$ & Number of platelet subcompartments & 9 & $\rightarrow$ & 9 & $\begin{array}{l}\text { Dowling et al. (2010); } \\
\text { Scholz et al. (2010) }\end{array}$ \\
\hline$\theta_{2}$ & $\begin{array}{l}\text { Decay rate of mediator due to } x_{2} \text { cells rel- } \\
\text { ative to decay rate due to } x_{1} \text { cells }\end{array}$ & 0.03 & Optimized & 0.006 & Optimized \\
\hline$\theta_{3}$ & $\begin{array}{l}\text { Decay rate of mediator due to } x_{3} \text { cells rel- } \\
\text { ative to decay rate due to } x_{1} \text { cells }\end{array}$ & $0.03 \cdot \theta_{2}$ & $\rightarrow$ & $0.03 \cdot \theta_{2}$ & $\begin{array}{l}\text { Kuwaki et al. (1998); } \\
\text { Broudy et al. (1997) }\end{array}$ \\
\hline$\tau_{\max }$ & Maximum MK maturation time & $5 /\left(\delta_{0} \cdot 4\right)$ & Assumed & $5 /\left(\delta_{0} \cdot 4\right)$ & Assumed \\
\hline$\tau_{\min }$ & Minimum MK maturation time & $3 /\left(\delta_{0} \cdot 4\right)$ & Assumed & $3 /\left(\delta_{0} \cdot 4\right)$ & Assumed \\
\hline$\lambda$ & $\begin{array}{l}\text { Strength of } x_{3} \text { feedback on immature MK } \\
\text { maturation }\end{array}$ & 7.32 & Optimized & 3.5 & Optimized \\
\hline$\sigma$ & Number of platelets per MK & 3000 & $\rightarrow$ & 3000 & $\begin{array}{l}\text { Smirnova (2010); Deutsch } \\
\text { and Tomer (2013) }\end{array}$ \\
\hline$D_{1}^{0}$ & $\begin{array}{l}\text { Determines fraction of } x_{1} \text { targets that are } \\
\text { hit by radiation at dose } D\end{array}$ & $1.18 \mathrm{~Gy}$ & $\begin{array}{l}\text { Hrodin et al. } \\
(2007)\end{array}$ & $0.79 \mathrm{~Gy}$ & Kashiwakura et al. (2000) \\
\hline$n_{1}$ & Number of targets per $x_{1}$ cell & 1 & $\rightarrow$ & 1 & Kashiwakura et al. (2000) \\
\hline$D_{2}^{0}$ & $\begin{array}{l}\text { Determines fraction of } x_{2} \text { targets that are } \\
\text { hit by radiation at dose } D\end{array}$ & $49.20 \mathrm{~Gy}$ & Optimized & $2.77 \mathrm{~Gy}$ & Optimized \\
\hline$n_{2}$ & Number of targets per $x_{2}$ cell & 4 & Assumed & 4 & Assumed \\
\hline$\mu$ & Rate of damaged cell death & $1.0 d^{-1}$ & $\rightarrow$ & $1.0 d^{-1}$ & Smirnova (2010) \\
\hline
\end{tabular}

$\rightarrow$ assumed to equal value in human model

supported by experimental in vitro data in human cells (Monzen et al., 2009). However, further experiments are warranted to both validate this proposed mechanism and explain the differences in MK radiosensitivity across species.

Our model successfully reproduces the platelet count nadirs, which occur within 10 to 20 days following radiation exposure for the rhesus model and 20 to 30 days following radiation exposure for the human model. The difference in time of nadir is due to species-dependent MK maturation times and platelet lifespans. The longer platelet lifespan and MK maturation time in humans leads to a later nadir than that seen in rhesus.

The recovery characteristics of the model are affected by a combination of parameters. In the human model, platelet recovery is characterized by a distinct rebounding during which the platelet concentration increases above normal pre-radiation levels which is in agreement with data from exposed individuals. This rebound is not observed in the rhesus model. This is partially due to the larger value of $\gamma$ in the human model $\left(0.26 d^{-1}\right)$ compared with the rhesus model $\left(0.06 d^{-1}\right)$. When $\gamma$ was reduced in the human model, the rebound was not observed. The accelerated recovery in the human model compared with the rhesus model could also be the result of treatment confounding the results.

It is clear from these comparisons that while both models perform very well, the rhesus model fits experimental results better than the human model. This is to be ex- pected since the animal data is collected under more controlled and precise experimental conditions. Additional sources of uncertainty and variability in human observational data that do not exist in the rhesus experimental data include uncertainty in the estimated dose, other dose types (neutron and internal contamination), protracted effects (i.e. lower does rate over longer durations), nonuniformity of the exposure, treatment, and inter-individual variability. Some subjects with a higher estimated radiation dose have a less intense response than subjects with lower radiation doses. For example, the 2.6 Gy case study from Goans (2012) had a lower normalized thrombocyte nadir when compared with the 3.51 Gy Vinca case study B (Jammet et al., 1959; Pei, 2012). This inconsistency could arise from individual variability in the response to radiation or result from uncertainty in the reported dose. Such discrepancies in the case study data help explain why some of the model predictions vary significantly from observational data.

In some case studies included in the optimizations, treatment details were not provided (Goans, 2012; Yarmonenko, 1988). When comparing simulations to data from subjects that had known treatment, earlier recovery than that predicted by the model was observed (Math et al., 1964). Thus, treatment may have large effects on the thrombopoietic system, and uncertainties in treatment could be affecting model comparisons as well as resulting optimized parameter values. Partial body exposures also re- 

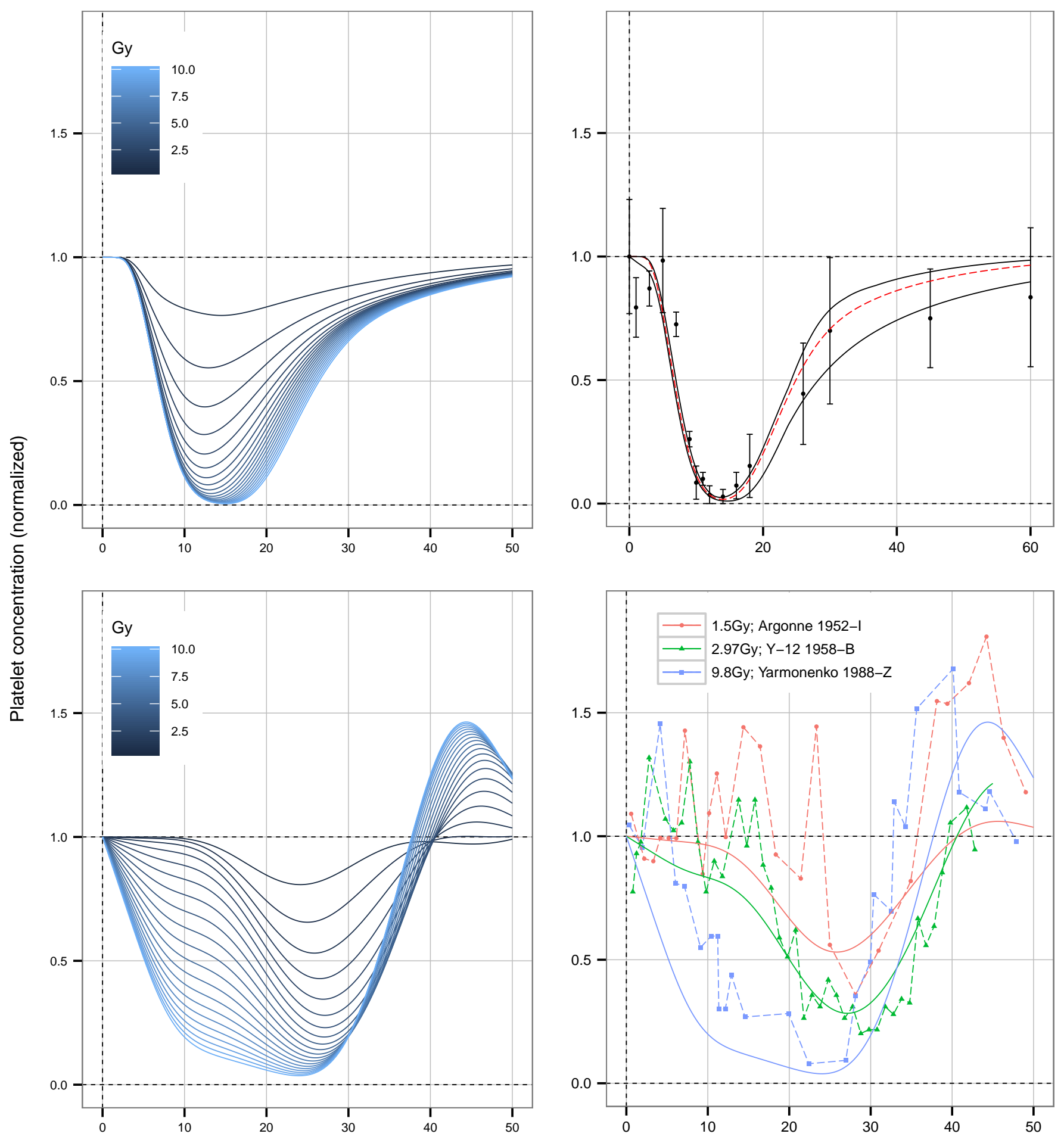

Time following radiation (days)

Figure 3: Thrombopoiesis model matches observed trends. Top Left: Rhesus thrombopoiesis simulations at increasing dose levels. Top Right: Rhesus data following 7 Gy irradiation (data obtained from Gluzman-Poltorak et al. (2014a) and Neumedicines, Inc.) overlaid on thrombopoiesis simulations (includes range based on sampling of parameter values from MCMC analysis). Bottom Left: Human thrombopoiesis simulations at increasing dose levels. Bottom Right: Human case study data (Mettler et al., 2001; Yarmonenko, 1988) overlaid on thrombopoiesis simulations.

sult in deviations from the simulation predictions (Subject K from Yarmonenko (1988)). For both treatment and partial body exposure, more analyses would need to be conducted to determine if deviations from the model are 


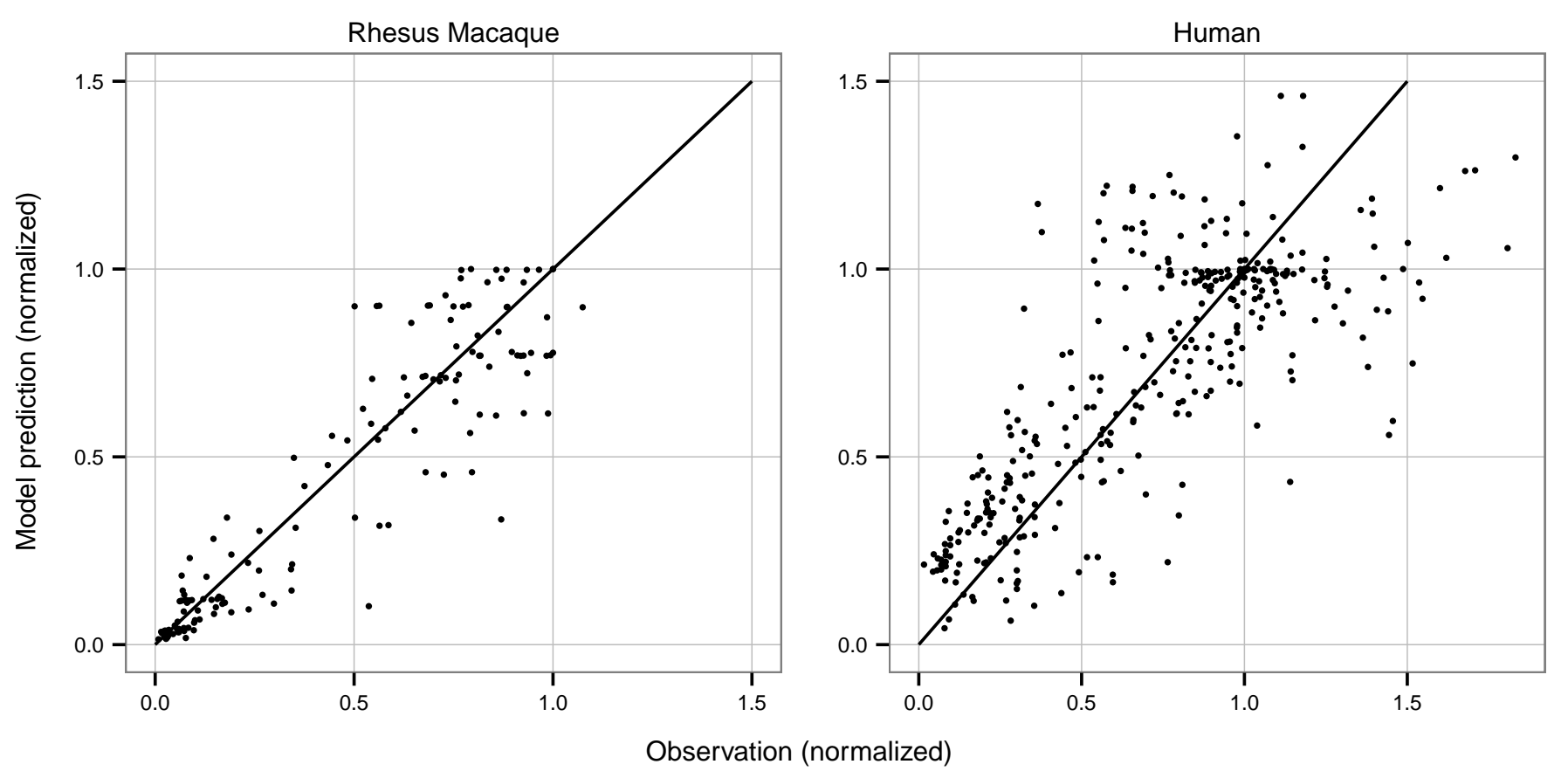

Figure 4: Assessing the quality of the rhesus and human models by comparing observations used for optimization with model predictions. Left Panel: Rhesus model/data (Basile et al., 2012; Gluzman-Poltorak et al., 2014a,b; Authier et al., 2013; Neumedicines, Inc.); Right Panel: Human model/data (Hempelmann et al., 1952; Andrews et al., 1961; Bond et al., 1965; Jammet et al., 1959; Yarmonenko, 1988; Baranov et al., 1994; Miller et al., 1958).

also in part due to other factors such as dose uncertainty, individual variability, and model structure error.

Given the inherent uncertainties and variability in available human radiation accident data, the degree of accuracy achieved by the presented model is quite remarkable.

\subsection{Validation of thrombopoiesis model using platelet $d y$ - namic data and compartment concentrations}

The thrombopoiesis models were validated using three independent techniques. First, model outputs were compared to platelet dynamic data from rhesus and human radiation exposures not used in the optimizations (Rhesus: Haigh and Paterson (1956); Farese et al. (1993); Burdelya et al. (2008); Gluzman-Poltorak et al. (2014b); Human: Hempelmann et al. (1952); Bond et al. (1965); Hirama et al. (2003); Stavem et al. (1985); Mettler et al. (2001); Mettler (2001)). Second, model output was compared to platelet dynamic data from thrombopheresis patients (Lasky et al., 1981; Weisbach et al., 1999; Sullivan et al., 1977). Third, the relative compartment sizes calculated using experimental data were compared to the relative compartment sizes calculated using model parameters.

Select results of the validation comparing model output with platelet dynamic data following irradiation not included in the optimization are shown in Figure 5. An observed verse predicted comparison for all the radiation validation data is shown in Figure 6. Similar to the optimization results, the models are able to accurately predict the observed trends in platelet concentration following radiation exposure.

The thrombopheresis validation results are shown in Figure 7. In thrombopheresis a certain percentage of platelets is removed from the blood stream. We can initialize the value of $x_{3}$ in our model using this percentage, where we assume (1) an equal fraction of platelets is removed from each age dependent platelet sub-compartment and (2) the removal of platelets is instantaneous. In one study, thrombopheresis was performed twice. To simulate this, we initialized the value of $x_{3}$ according to the first thrombopheresis and manually altered $x_{3}$ at the time of the second thrombopheresis without changing the values of the other state variables. This validation helps to confirm that the kinetic rates of cell maturation and feedback are correct in the model. In four separate studies, two of which had mean data from multiple subjects, the model is able to accurately predict the platelet count recovery following platelet removal. These comparisons reassure us that the kinetic parameters obtained from the literature and through optimization are accurate.

To further validate the thrombopoiesis model, the relative compartment sizes under steady-state conditions are calculated using model parameters and compared to compartment sizes predicted using experimental data. For the calculation of the relative compartment sizes using parameter values see the Appendix A.2. To perform this comparison, the total number of platelets and MKs was determined as a function of $\mathrm{kg}$ body weight. Table 3 compares 

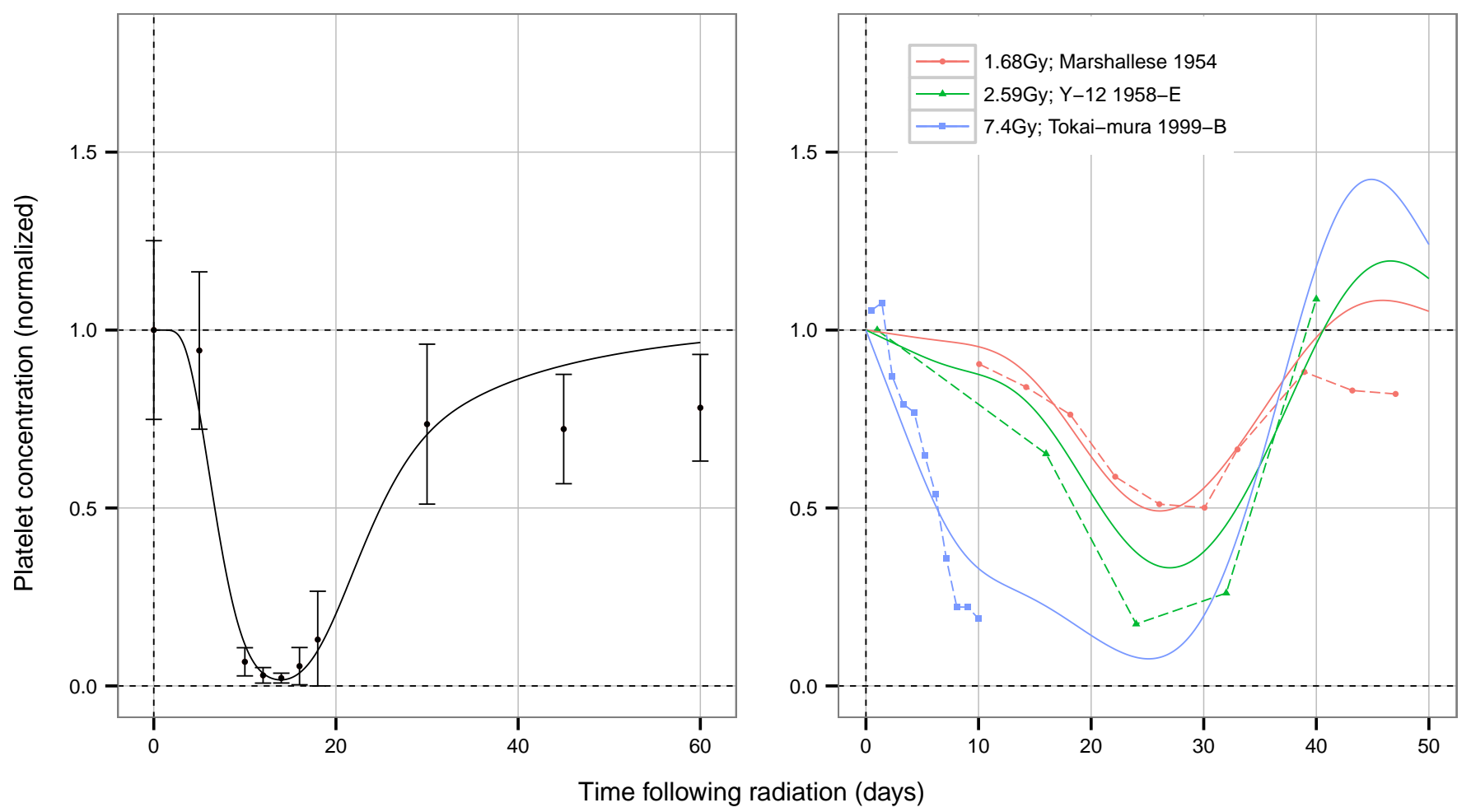

Figure 5: Thrombopoiesis model accurately predicts trends in data not used for optimization. Left: Rhesus validation data following 7 Gy irradiation (Gluzman-Poltorak et al., 2014b). Right: Human case studies reserved for validation compared to thrombopoiesis model simulation at specified doses.

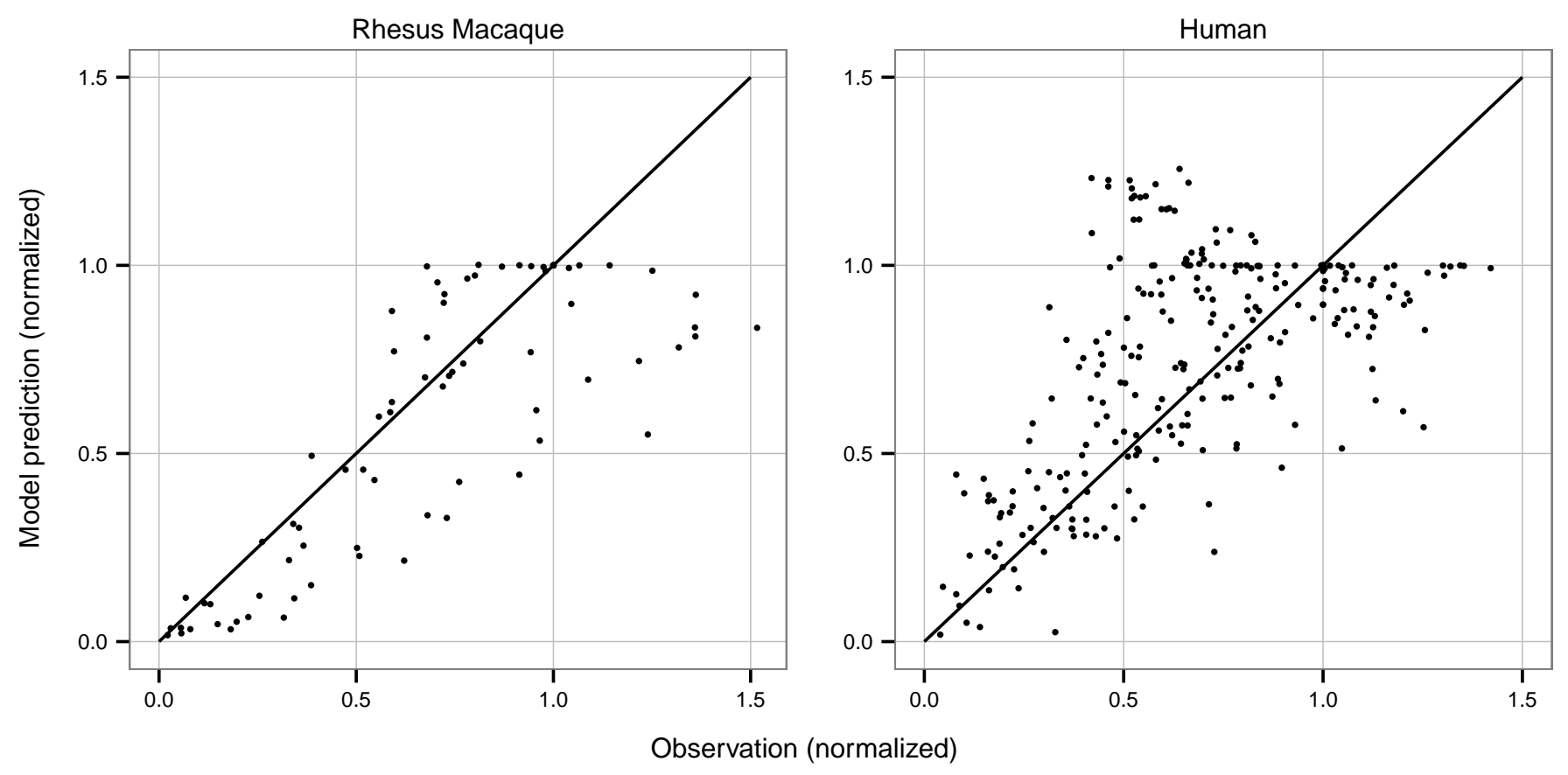

Figure 6: Assessing the quality of the rhesus and human models by comparing observations used for validation with model predictions. Left Panel: Rhesus model/data (Haigh and Paterson, 1956; Farese et al., 1993; Burdelya et al., 2008; Gluzman-Poltorak et al., 2014b); Right Panel: Human model/data (Hempelmann et al., 1952; Bond et al., 1965; Hirama et al., 2003; Stavem et al., 1985; Mettler et al., 2001; Mettler, 2001). 

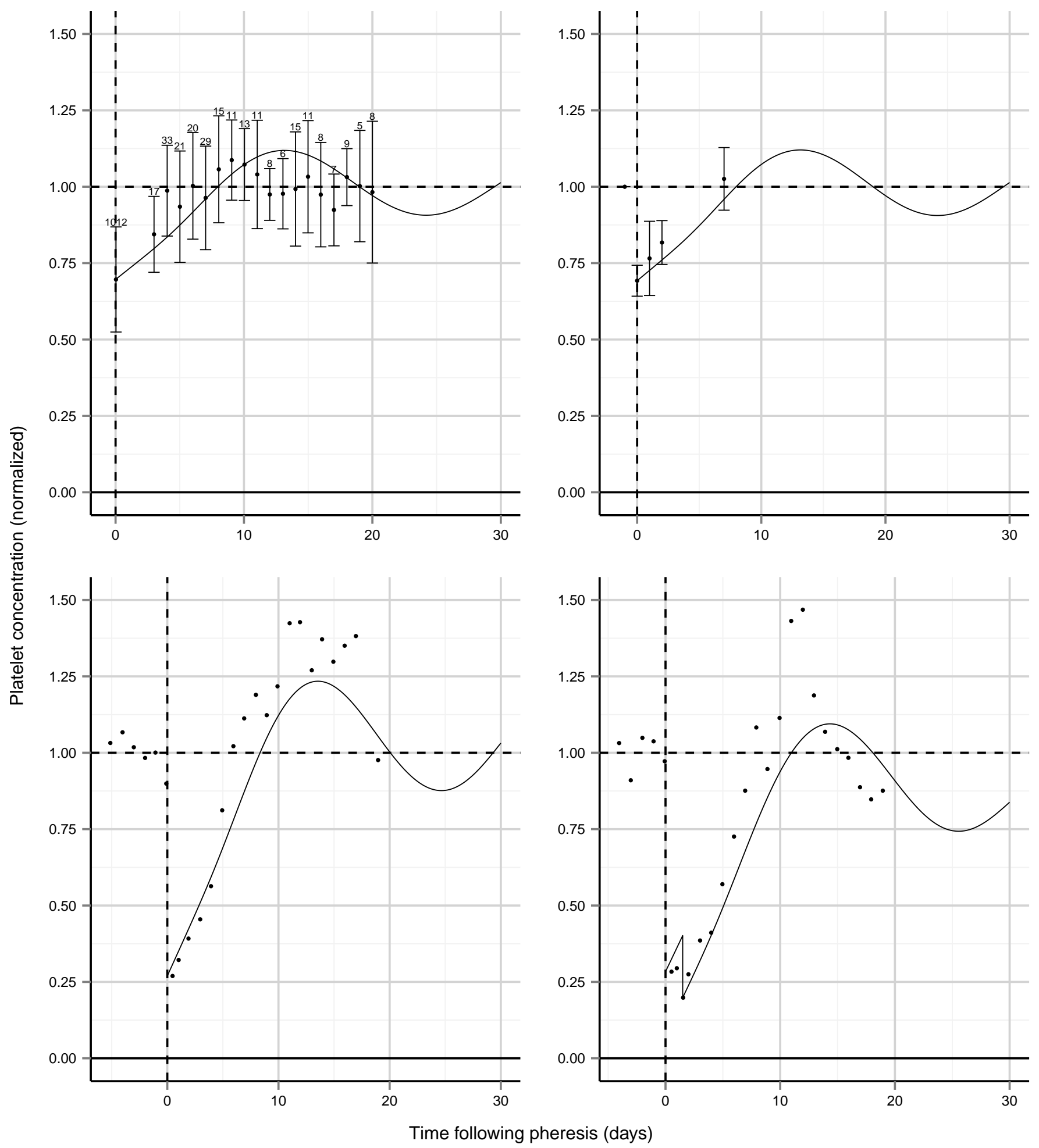

Figure 7: Thrombopoiesis model overlaid on thrombopheresis data. Top left: Data from Lasky et al. (1981), the number above each data point specifies the number of subjects (mean \pm SEM). Top right: Data from Weisbach et al. (1999), data points from 22 subjects (mean \pm SD). Bottom left: Individual data from Sullivan et al. (1977). Bottom right: Individual data from Sullivan et al. (1977), subject underwent two platelet removal procedures.

the relative size of the platelet and MK compartments as determined with experimental predictions and model parameters. For the human model, the model prediction is approximately equal to the experimental prediction. For rhesus, the model prediction of the ratio of platelets to MKs appears greater than the experimental prediction. 
However, the errors on some of the variables are not available (see Table 3); therefore, the true range for the experimental prediction cannot be calculated. Furthermore, the value of platelets produced per MK, $\sigma$, is set to 3000 ; however, some sources suggest that this value can range from 1000 to 3000 (Deutsch and Tomer, 2013). Based on this range of uncertainty, the model prediction is in reasonable agreement with the experimental prediction.

\subsection{Simulations are Consistent with METREPOL and UN- SCEAR}

Medical Treatment Protocols for Radiation Accident Victims as a Basis for a Computerised Guidance System (METREPOL) provides an assessment of radiation injury severity based upon the hematopoietic response in humans (Fliedner et al., 2001). This severity ranking system was developed by an international group of selected experts and is based on both experimental data and the analysis of data from radiation victims. There are four possible severity grades (H1: Mild damage; H2: Moderate damage; H3: Severe damage; H4: Fatal damage) which are not based on radiation dose but on the ensuing symptoms.

To verify that our human thrombopoiesis model is in agreement with the predicted hematopoietic responses from METREPOL, we developed overlays of our model on the METREPOL predictions for the H2 and H3 severity levels (Figure 8). For an accurate comparison, the thrombopoietic model output is shown for the normal range of blood cell counts as given in the METREPOL report (Fliedner et al., 2001). Thrombopoiesis simulations for 4 and 7 Gy correlate well with METREPOL H2 and H3 severity categories as shown in Figure 8. Overall, the model predictions match the trends given by METREPOL. The platelet recovery predicted by our model is slightly faster and leads to an overshoot not predicted in the METREPOL hematopoietic response; however, this overshoot is observed in several of the individual case studies examined in our work (See Figure 3).

In a second set of expert predictions, Annex G of the United Nations Scientific Committee on the Effects of Atomic Radiation (UNSCEAR) 1988 report presents a schematic picture of the average time course of blood cell counts deduced from accidental human exposures (UNSCEAR, 1988). Figure 9 shows a comparison of our human thrombopoiesis model output with this expert prediction. The UNSCEAR report presents the predicted blood cell responses at doses ranging from 0 to 20 Gy divided into four groups. Therefore, we plot our hematopoietic model outputs as a shaded region based on each dose range and overlay the response predicted by UNSCEAR. Although the model is not in exact agreement with the UNSCEAR predictions, the general trends are supported and the dose response matches fairly well. Our model predicts a slightly faster recovery than expert predictions.

\subsection{Application of model: Prediction of treatment require- ments}

One example application of the model is to predict treatment requirements of a population following a nuclear event. The purpose of the human thrombopoiesis model is to capture the average platelet dynamics following radiation exposure. By running Monte Carlo simulations, we can predict the response distribution for a large population and, in turn, predict treatment requirements. As a simplified example, we assume the one source of variability in the population is the initial platelet concentration and run a Monte Carlo simulation to simulate the platelet response in 400 individuals following a set dose of 6 Gy (Figure 10). Using this response distribution, we can predict the number of transfusions required only once per individual as a function of time following the exposure where we assume transfusions are required when platelet levels decline below $20 \cdot 10^{3}$ cells per $\mu L$.

Although this is clearly a simplified example, it provides the framework for how a model of thrombopoiesis following radiation exposure can be used to assist in disaster preparedness. Other endpoints, such as survivability can also be examined. The depth of the platelet nadir and the rate of decline have been correlated with the probability of survival (Housinger et al., 1993; Akca et al., 2002; Wang et al., 2010). Thus, similar approaches could be used in which distributions for other features (i.e., nadir, initial

Table 3: Comparison of model and experimental prediction of relative size of platelet and MK compartment.

\begin{tabular}{lll}
\hline & Rhesus & Human \\
\hline BMC $\left(10^{10} / \mathrm{kg}\right)$ & $2.4($ Pegg, 1966) & 2 (Valentin, 2002) \\
MK (\% of BMC) & $0.03($ Usacheva and Raeva, 1963) & $0.04 \pm 0.01$ (Levine, 1980) \\
Plts in Blood $\left(10^{3} / \mu L\right)$ & $373.66 \pm 92.62$ (Usacheva and Raeva, 1963) & 250 (Valentin, 2002) \\
Blood Volume $(\mathrm{mL} / \mathrm{kg})$ & 54 (Drexel University College of Medicine, 2011) & $67.5 \pm 3.5$ (Starr and Taggart, 1989; \\
& & World Book Inc. Staff, 1998) \\
Predictions (Plts/MK) & & \\
Experimental* & $2802 \pm 695$ & $2109 \pm 539$ \\
Model $^{* *}$ & 4333 & 1636 \\
\hline
\end{tabular}

BMC: Bone Marrow Cells

Plts: Platelets

*Experimental Prediction=Platelets in Blood · Blood Volume/(BM Cells $\cdot$ MK/100)

**For model prediction calculation see Appendix A.2. 


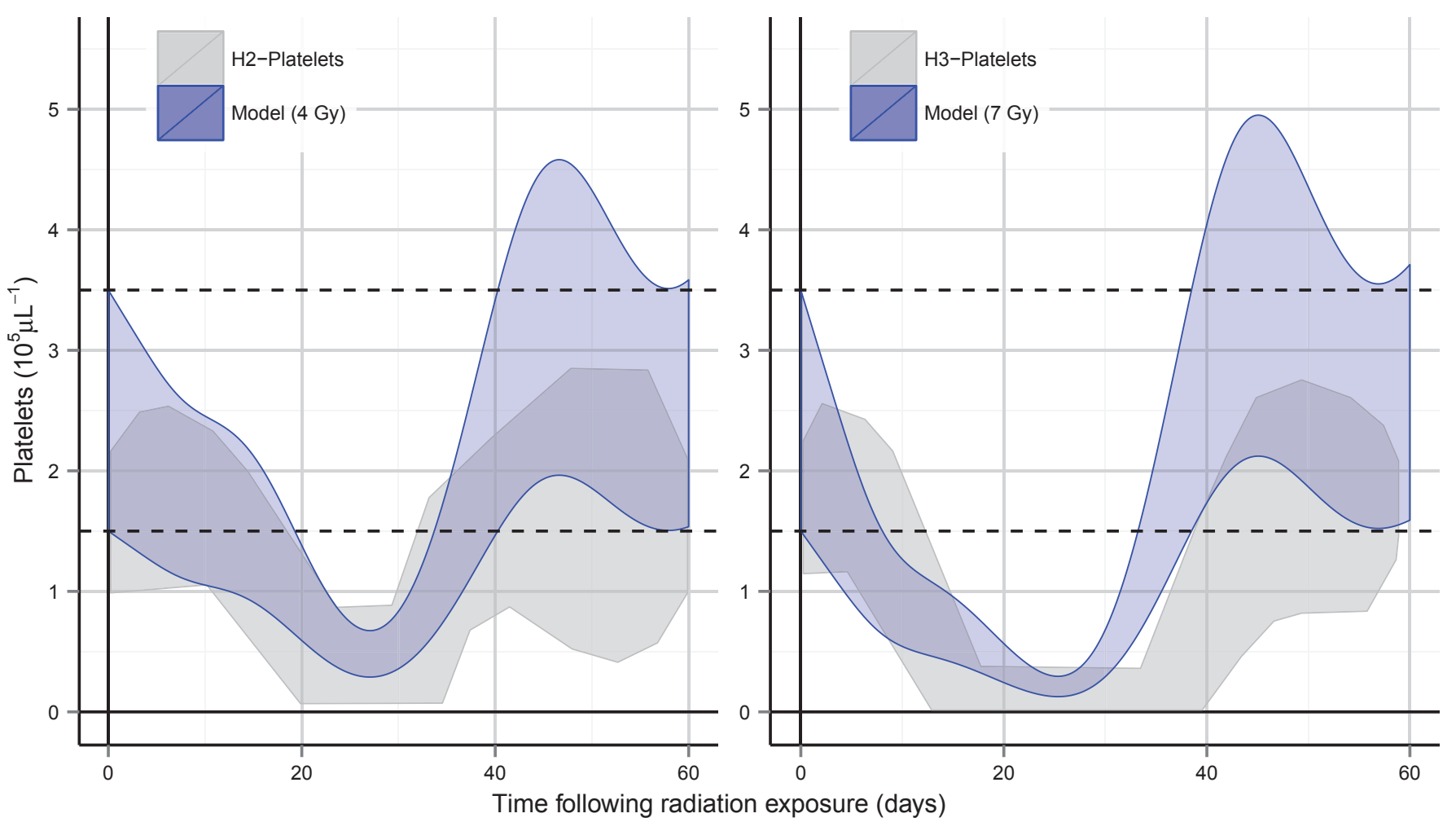

Figure 8: Comparison of thrombopoiesis model output to METREPOL predictions (Fliedner et al., 2001).

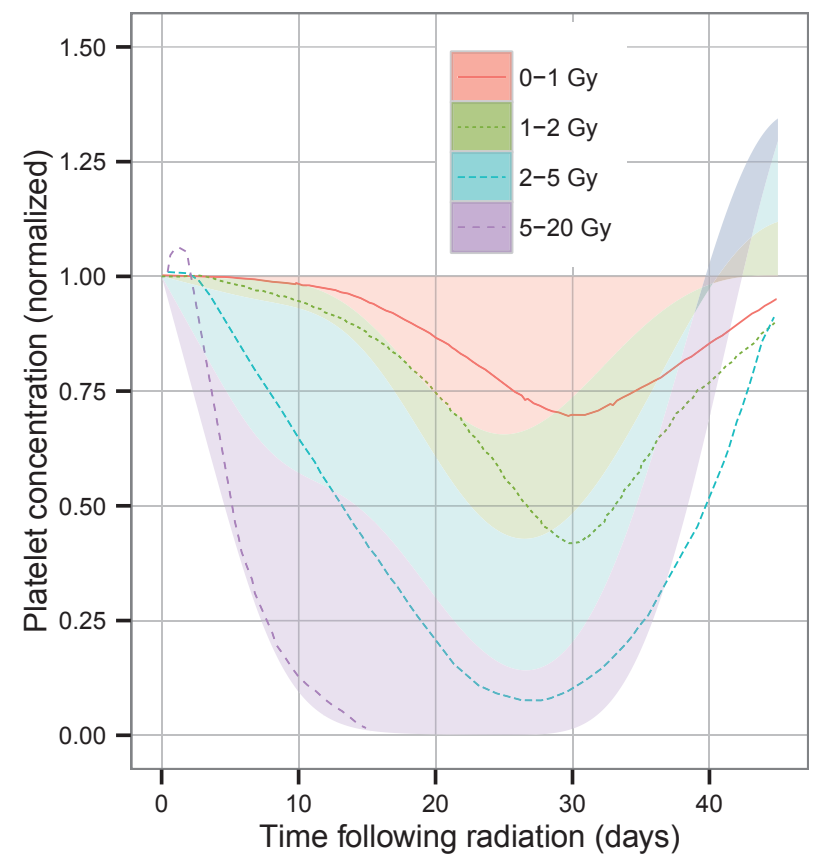

Figure 9: Comparison of thrombopoiesis model output to expert predictions. Model output shown as shaded region for each dose range and expert predictions from UNSCEAR report shown as solid/dashed lines (UNSCEAR, 1988) . rate of decline, duration of cytopenia) are analyzed as a function of radiation dose using Monte Carlo simulations. Future work would involve correlating model outputs with survival.

\section{Conclusion}

We have developed a model of thrombopoiesis following acute radiation exposure in rhesus macaques and humans that provides realistic predictions on platelet dynamics, capturing specific dose-dependent trends. While large variances exist in human case study data, the goal of the model is to capture the average response within a population. This model can eventually be used to assist in disaster preparedness. Furthermore, the mechanistic models allow us to extrapolate experimental observations in rhesus to predict effects in humans.

Throughout model development, several assumptions were made regarding parameter values due to a lack of experimental data. Future work would involve replacing these assumed values with empirically derived values as more data becomes available. This would enhance the biological meaning of the parameter values, and, in turn, allow more accurate predictions of how perturbations to biological kinetic rates would effect the system. 

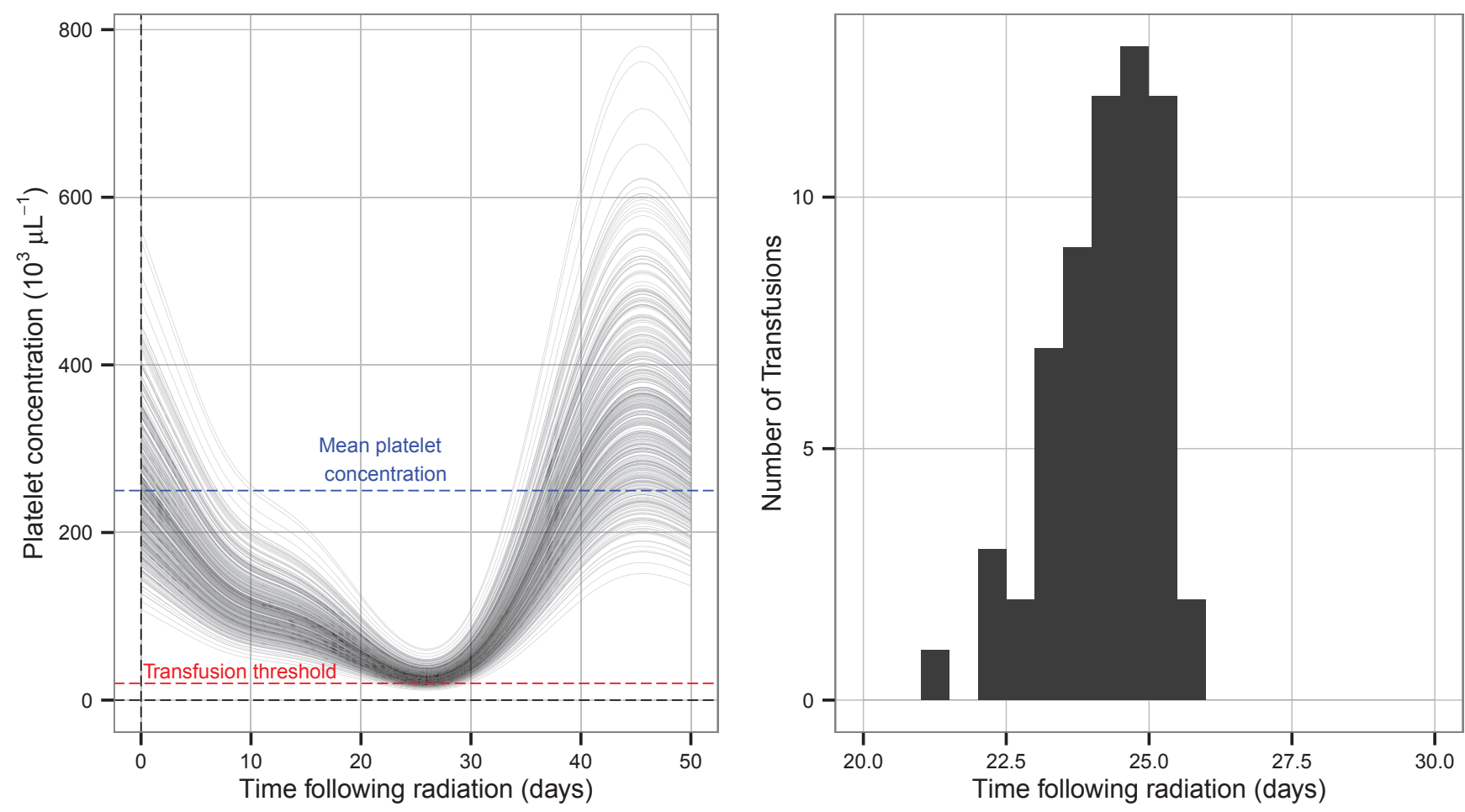

Figure 10: Estimation of population treatment requirements following 6 Gy radiation exposure. Left: 400 simulations for which each simulation used a baseline platelet count randomly sampled from a log normal distribution describing platelet concentration in healthy humans based on a $95 \%$ confidence interval of 140-250 cells $10^{3} \mu L^{-1}$ (Valentin, 2002). Right: Histogram of transfusions needed as a function of time, assuming one transfusion per patient for platelet counts $\leq 20$ cells $10^{3} \mu L^{-1}$.

\section{Acknowledgements}

Funding for this work was provided by the Defense Threat Reduction Agency under Contract HDTRA1-14-D0003-0005 and by the Biomedical Advanced Research and Development Authority, Office of the Assistant Secretary for Preparedness and Response, Office of the Secretary, Department of Health and Human Services, under Contract Numbers HHSO100201100037C and HHSO100200800060

\section{References}

S. Akca, P. Haji-Michael, A. de Mendona, P. Suter, M. Levi, and J. L. Vincent. Time course of platelet counts in critically ill patients. Critical care medicine, 30(4):753-6, 04 2002. ISSN 0090-3493. URL http://www.ncbi.nlm.nih.gov/pubmed/11940740.

M. Andreeff, D. W. Goodrich, and A. B. Pardee. Cell proliferation, differentiation, and apoptosis. In R. C. Bast, D. W. Kufe, R. E. Pollock, R. R. Weichselbaum, J. F. Holland, and E. Frei, editors, Holland-Frei Cancer Medicine, 5th edition. BC Decker, Ontario, Canada, 2000.

G. A. Andrews, B. W. Sitterson, A. L. Kretchmar, and M. Brucer. Criticality accident at the Y-12 plant. Diagnosis and Treatment of Acute Radiation Injury, pages 27-48, 1961.

S. Authier, M. Pouliot, A. Ascah, E. Troncy, and R. Forster. Hematopoietic characterization of a non-rodent model for acute radiation syndrome, 2013. URL http://www.citoxlab.com/wpcontent/uploads/2014/03/28-2012_SOT_Acute-radiationsyndrome-hematopoietic-characterization-of-a-non-rodentmodel.pdf.
A. E. Baranov, G. D. Selidovkin, A. Butturini, and R. P. Gale. Hematopoietic recovery after $10-$ Gy acute total body radiation. Blood, 83(2):596-599, 01 1994. ISSN 0006-4971. URL http: //www.ncbi.nlm.nih.gov/pubmed/8286754.

L. A. Basile, D. Ellefson, Z. Gluzman-Poltorak, K. Junes-Gill, V. Mar, S. Mendonca, J. D. Miller, J. Tom, A. Trinh, and T. K. Gallaher. HemaMax, a recombinant human interleukin-12, is a potent mitigator of acute radiation injury in mice and non-human primates. PloS one, 7(2):e30434, 01 2012. ISSN 1932-6203. doi: 10.1371/journal.pone.0030434. URL http://www.ncbi.nlm.nih.

.gov/pubmed/22383962.

V. P. Bond, T. M. Fliedner, and J. O. Archambeau. Mammalian radiation lethality: A disturbance in cellular kinetics. Academic Press, New York and London, 1965. URL http://www.getcited. org/pub/101214105.

V. C. Broudy, N. L. Lin, D. F. Sabath, T. Papayannopoulou, and K. Kaushansky. Human platelets display high-affinity receptors for thrombopoietin. Blood, 89(6):1896-904, 03 1997. ISSN 00064971. URL http://www.ncbi.nlm.nih.gov/pubmed/9058709.

R. Brun, P. Reichert, and H. R. Knsch. Practical identifiability analysis of large environmental simulation models. Water Resources Research, 37(4):1015-1030, 04 2001. ISSN 00431397. doi: 10.1029/2000WR900350. URL http://doi.wiley.com/10.1029/ 2000WR900350.

L. G. Burdelya, V. I. Krivokrysenko, T. C. Tallant, E. Strom, A. S. Gleiberman, D. Gupta, O. V. Kurnasov, F. L. Fort, A. L. Osterman, J. a. Didonato, E. Feinstein, and A. V. Gudkov. An agonist of toll-like receptor 5 has radioprotective activity in mouse and primate models. Science (New York, N.Y.), 320(5873):226-30, 04 2008. ISSN 1095-9203. doi: 10.1126/science.1154986. URL http://www.ncbi.nlm.nih.gov/pubmed/18403709.

V. R. Deutsch and A. Tomer. Advances in megakaryocytopoiesis and thrombopoiesis: From bench to bedside. British Journal 
of Haematology, 161(6):778-793, 06 2013. ISSN 1365-2141. doi: 10.1111/bjh.12328. URL http://www.ncbi.nlm.nih.gov/pubmed/ 23594368.

M. R. Dowling, E. C. Josefsson, K. J. Henley, P. D. Hodgkin, and B. T. Kile. Platelet senescence is regulated by an internal timer not damage inflicted by hits. Blood, 116(10):1776-1778, 092010. ISSN 1528-0020. doi: 10.1182/blood-2009-12-259663. URL http: //www.ncbi.nlm.nih.gov/pubmed/20530288.

Drexel University College of Medicine. A compendium of drugs used for laboratory animal anesthesia, analgesia, tranquilization and restraint, 2011. URL www.drexelmed.edu/documents/ULAR/IACUC_ drugs.pdf.

S. Ebbe, F. Stohlman, J. Donovan, and J. Overcash. Megakaryocyte maturation rate in thrombocytopenic rats. Blood, 32(5):787-795, 11 1968. ISSN 0006-4971. URL http://www.ncbi.nlm.nih.gov/ pubmed/5687938.

A. M. Farese, D. E. Williams, F. R. Seiler, and T. J. MacVittie Combination protocols of cytokine therapy with interleukin-3 and granulocyte-macrophage colony-stimulating factor in a primate model of radiation-induced marrow aplasia. Blood, 82(10):30123018, 11 1993. ISSN 0006-4971. URL http://www.ncbi.nlm.nih. gov/pubmed/8219192

C. A. Finch, L. A. Harker, and J. D. Cook. Kinetics of the formed elements of human blood. Blood, 50(4):699-707, 10 1977. ISSN 0006-4971. URL http://www.ncbi.nlm.nih.gov/pubmed/332255.

T. Fliedner, I. Friesecke, and K. Beyrer, editors. Medical management of radiation accidents: manual on the acute radiation syndrome. British Institute of Radiology, 2001. ISBN 0905749464.

Z. Gluzman-Poltorak, S. R. Mendonca, V. Vainstein, H. Kha, and L. A. Basile. Randomized comparison of single dose of recombinant human IL-12 versus placebo for restoration of hematopoiesis and improved survival in rhesus monkeys exposed to lethal radiation. Journal of hematology $\&$ oncology, 7:31, 01 2014a. ISSN 1756-8722. doi: 10.1186/1756-8722-7-31. URL http://www.pubmedcentral.nih.gov/articlerender.fcgi? artid=4108131\&tool=pmcentrez\&rendertype=abstract

Z. Gluzman-Poltorak, V. Vainstein, and L. A. Basile. Recombinant interleukin-12, but not granulocyte-colony stimulating factor, improves survival in lethally irradiated nonhuman primates in the absence of supportive care: Evidence for the development of a frontline radiation medical countermeasure. American journal of hematology, 89(9):868-73, 09 2014b. ISSN 1096-8652. doi: 10.1002/ajh.23770. URL http://www.ncbi.nlm.nih.gov/pubmed/ 24852354.

R. E. Goans. Radiation exposure, illness, and injury, 2012. URL http://www.publichealthontario.ca/en/ LearningAndDevelopment/Events/Documents/EMS2-Feb.24, 2012 . pdf.

D. H. Graessle. Simulation of Radiation Effects Using Biomathematical Models of the Megakaryocytic Cell Renewal System. PhD thesis, Medizinische Fakultt der Universitt Ulm, 2000. URL http://www.researchgate.net/publication/29528258_ Simulation_of_radiation_effects_using_biomathematical_ models_of_the_megakaryocytic_cell_renewal_system/file/ 79e4150c662224a547.pdf.

M. L. Greenberg, A. D. Chanana, E. P. Cronkite, L. M. Schiffer, and P. A. Stryckmans. Extracorporeal irradiation of blood in man: radiation resistance of circulating platelets. Radiation research, 35(1):147-54, 07 1968. ISSN 0033-7587. URL http://www.rrjournal.org/doi/abs/10.2307/ 3572441http://www.ncbi.nlm.nih.gov/pubmed/5241847.

H. Haario, E. Saksman, and J. Tamminen. An adaptive Metropolis algorithm. Bernoulli, 7(2):223-242, 2001.

M. V. Haigh and E. Paterson. Effects of a single session of whole body irradiation in the rhesus monkey. The British journal of radiology, 29(339):148-57, 03 1956. ISSN 0007-1285. URL http: //www.ncbi.nlm.nih.gov/pubmed/13304287.

L. A. Harker, U. M. Marzec, A. B. Kelly, E. Cheung, A. Tomer, J. L. Nichol, S. R. Hanson, and R. B. Stead. Prevention of thrombocytopenia and neutropenia in a nonhuman primate model of marrow suppressive chemotherapy by combining pegylated re- combinant human megakaryocyte growth and development factor and recombinant human granulocyte colony-stimulating factor. Blood, 89(1):155-65, 01 1997. ISSN 0006-4971. URL http://www.ncbi.nlm.nih.gov/pubmed/8978288.

L. A. Harker, L. K. Roskos, U. M. Marzec, R. A. Carter, J. K. Cherry, B. Sundell, E. N. Cheung, D. Terry, and W. Sheridan. Effects of megakaryocyte growth and development factor on platelet production, platelet life span, and platelet function in healthy human volunteers. Blood, 95(8):25142522, 2000. URL http://bloodjournal.hematologylibrary.org/ content/95/8/2514. short.

L. H. Hempelmann, H. Lisco, and J. G. Hoffman. The acute radiation syndrome: a study of nine cases and a review of the problem. Annals of internal medicine, 36(2:1):279-510, 02 1952. ISSN 00034819. URL http://www.ncbi.nlm.nih.gov/pubmed/14895043.

D. Heylmann, F. Rdel, T. Kindler, and B. Kaina. Radiation sensitivity of human and murine peripheral blood lymphocytes, stem and progenitor cells. Biochimica et biophysica acta, 1846(1):121-9, 08 2014. ISSN 0006-3002. doi: 10.1016/j.bbcan.2014.04.009. URL http://www.ncbi.nlm.nih.gov/pubmed/24797212.

T. Hirama, S. Tanosaki, S. Kandatsu, N. Kuroiwa, T. Kamada, H. Tsuji, S. Yamada, H. Katoh, N. Yamamoto, H. Tsujii, G. Suzuki, and M. Akashi. Initial medical management of patients severely irradiated in the Tokai-mura criticality accident. The British Journal of Radiology, 76(904):246-253, 04 2003. ISSN 0007-1285. doi: 10.1259/bjr/82373369. URL http: //bjr.birjournals .org/cgi/doi/10.1259/bjr/82373369http: //www.birpublications.org/doi/abs/10.1259/bjr/82373369.

T. A. Housinger, C. Brinkerhoff, and G. D. Warden. The relationship between platelet count, sepsis, and survival in pediatric burn patients. Archives of Surgery, 128(1):65-7, 01 1993. ISSN 0004-0010. URL http://www.ncbi.nlm.nih.gov/pubmed/8418782.

F. Hrodin, L. Roy, N. Grenier, C. Delaunay, S. Baug, A. Vaurijoux, E. Grgoire, C. Martin, A. Alonso, J.-F. Mayol, and M. Drouet. Antiapoptotic cytokines in combination with pegfilgrastim soon after irradiation mitigates myelosuppression in nonhuman primates exposed to high irradiation dose. Experimental hematology, 35(8): 1172-81, 08 2007. ISSN 0301-472X. doi: 10.1016/j.exphem.2007. 04.017. URL http://www.ncbi.nlm.nih.gov/pubmed/17560010.

H. Jammet, G. Mathe, B. Pendic, J. F. Duplan, B. Maupin, R. Latarjet, D. Kalic, L. Schwarzenberg, Z. Djukic, and J. Vigna. Study of six cases of accidental acute total irradiation. Revue franaise d'tudes cliniques et biologiques, 4(3):210-25, 03 1959. ISSN 03704793. URL http://www.ncbi.nlm.nih.gov/pubmed/13646286.

M. C. Joiner. Quantifying cell kill and cell survival. In M. C. Joiner and A. van der Kogel, editors, Basic Clinical Radiobiology Fourth Edition, pages 42-55. CRC Press, 2009.

I. Kashiwakura, M. Kuwabara, O. Inanami, M. Murakami, Y. Hayase, T. A. Takahashi, and Y. Takagi. Radiation sensitivity of megakaryocyte colony-forming cells in human placental and umbilical cord blood. Radiation Research, 153(2):144-152, 02 2000. ISSN 0033-7587. URL http://www.ncbi.nlm.nih.gov/ pubmed/10629613.

K. Kato, A. Omori, and I. Kashiwakura. Radiosensitivity of human haematopoietic stem/progenitor cells. Journal of radiological protection: official journal of the Society for Radiological Protection, 33(1):71-80, 03 2013. ISSN 1361-6498. doi: 10.1088/0952-4746/ 33/1/71. URL http://www.ncbi.nlm.nih.gov/pubmed/23295782.

K. Kaushansky. The molecular mechanisms that control thrombopoiesis. The Journal of Clinical Investigation, 115(12): 3339-47, 12 2005. ISSN 0021-9738. doi: 10.1172/JCI26674. URL http://www.jci.org/cgi/content/abstract/115/12/3339http: //www.pubmedcentral.nih.gov/articlerender.fcgi?artid= 1297257\&tool=pmcentrez\&rendertype=abstract.

T. Kuwaki, T. Hagiwara, C. Yuki, I. Kodama, T. Kato, and H. Miyazaki. Quantitative analysis of thrombopoietin receptors on human megakaryocytes. FEBS letters, 427(1):46-50, 051998. ISSN 0014-5793. URL http://www.ncbi.nlm.nih.gov/pubmed/ 9613597.

M. Laine. Adaptive MCMC Methods with Applications in Environmental and Geophysical Models. Finish Meteorological Institute, 
2008.

L. C. Lasky, A. Lin, R. A. Kahn, and J. McCullough. Donor platelet response and product quality assurance in plateletpheresis. Transfusion, 21(3):247-260, 1981. ISSN 0041-1132. URL http://www.ncbi.nlm.nih.gov/pubmed/7233509.

R. F. Levine. Isolation and characterization of normal human megakaryocytes. British journal of haematology, 45(3):487-97, 07 1980. ISSN 0007-1048. URL http://onlinelibrary.wiley. com/doi/10.1111/j.1365-2141.1980.tb07168.x/abstracthttp: //www.ncbi.nlm.nih.gov/pubmed/7000150.

N. MacDonald. Time Lags in Biological Models. Lecture Notes in Biomathematics. Springer-Verlag, Berlin, 1978.

G. Math, J. L. Amiel, and L. Schwarzenberg. Treatment of acute total-body irradiation injury in man. Annals of the New York Academy of Sciences, 114(1):368-392, 1964. URL http://onlinelibrary.wiley.com/doi/10.1111/j. 1749-6632.1964.tb53591.x/full.

F. A. Mettler. Accidents in industrial radiation facilities. In I. A. Gusev, A. K. Guskova, and F. A. Mettler, editors, Medical Management of Radiation Accidents, pages 211-222. CRC Press, Boca Raton, 2 edition, 2001.

F. A. Mettler, B. L. Voelz, J. C. Nnot, and I. A. Gusev. Criticality accidents. In I. A. Gusev, A. K. Guskova, and F. A. Mettler, editors, Medical Management of Radiation Accidents, pages 173194. CRC Press, Boca Raton, 2 edition, 2001.

D. Mezzano, K. Hwang, and R. H. Aster. Characteristics of total platelet populations and of platelets isolated in platelet-rich plasma. Transfusion, 22(3):197-202, 1982. ISSN 0041-1132. URL http://www.ncbi.nlm.nih.gov/pubmed/7090031.

L. S. Miller, G. H. Fletcher, and H. B. Gerstner. Radiobiologic observations on cancer patients treated with whole-body x-irradiation. Radiation research, 8(2):150-65, 02 1958. ISSN 0033-7587. URL http://www.ncbi.nlm.nih.gov/pubmed/13506043.

S. Monzen, K. Osuda, Y. Miyazaki, N. Hayashi, K. Takahashi, and I. Kashiwakura. Radiation sensitivities in the terminal stages of megakaryocytic maturation and platelet production. Radiation research, 172(3):314-20, Sept. 2009. ISSN 0033-7587. doi: 10.1667/RR1519.1. URL http://www.ncbi.nlm.nih.gov/pubmed/ 19708780.

J. J. Mor. The Levenberg-Marquardt algorithm: Implementation and theory. In Numerical Analysis, pages 105-116. Springer Berlin Heidelberg, 1978.

E. A. Murphy and M. E. Francis. The estimation of blood platelet survival. II. The multiple hit model. Thrombosis et diathesis haemorrhagica, 25(1):53-80, 01 1971. ISSN 0340-5338. URL http://www.ncbi.nlm.nih.gov/pubmed/5577974.

D. E. Pegg. Bone marrow transplantation. Year Book Medical Publishers, Chicago, 1966.

M. Pei. Estimation of doses received by operators in the 1958 RB reactor accident using the MCNP5 computer code simulation. $\mathrm{Nu}$ clear Technology and Radiation Protection, 27(3):199-221, 2012. ISSN 1451-3994. doi: 10.2298/NTRP1203199P. URL http: //www.doiserbia.nb.rs/Article. aspx?ID=1451-39941203199P.

R Core Team. R: A Language and Environment for Statistical Computing. Technical report, R Foundation for Statistical Computing, Vienna, Austria, 2013. URL http://www.r-project.org/.

A. Schmitt, J. Guichard, J. M. Mass, N. Debili, and E. M. Cramer. Of mice and men: Comparison of the ultrastructure of megakaryocytes and platelets. Experimental Hematology, 29(11):12951302, 11 2001. ISSN 0301-472X. URL http://www.ncbi.nlm. nih.gov/pubmed/11698125.

M. Scholz, A. Gross, and M. Loeffler. A biomathematical model of human thrombopoiesis under chemotherapy. Journal of Theoretical Biology, 264(2):287-300, 05 2010. ISSN 1095-8541. doi: 10.1016/j.jtbi.2009.12.032. URL http://www.ncbi.nlm.nih.gov/ pubmed/20083124.

K. Skomorovski, H. Harpak, A. Ianovski, M. Vardi, T. P. Visser, S. C. C. Hartong, H. H. D. M. van Vliet, G. Wagemaker, and Z. Agur. New TPO treatment schedules of increased safety and efficacy: Pre-clinical validation of a thrombopoiesis simulation model. British Journal of Haematology, 123(4):683-91, 112003.
ISSN 0007-1048. URL http://www.ncbi.nlm.nih.gov/pubmed/ 14616973.

O. A. Smirnova. Environmental Radiation Effects on Mammals. Springer New York, New York, NY, 2010. ISBN 978-1-4419-72125. doi: 10.1007/978-1-4419-7213-2. URL http://link.springer. $\mathrm{com} / 10.1007 / 978-1-4419-7213-2$

O. A. Smirnova. Comparative analysis of the dynamics of thrombocytopoietic, granulocytopoietic, and erythropoietic systems in irradiated humans: a modeling approach. Health physics, 103(6):787-801, 12 2012. ISSN 1538-5159. doi: 10.1097/ HP.0b013e31826021bb. URL http://www.ncbi.nlm.nih.gov/ pubmed/23111526.

K. Soetaert and T. Petzoldt. Inverse modelling, sensitivity and Monte Carlo analysis in R using package FME. Journal of Statistical Software, 33(3):1-28, 2010. URL http://www.jstatsoft. org/v33/i03/

K. Soetaert, T. Petzoldt, and R. W. Setzer. Solving differential equations in R: Package deSolve. Journal of Statistical Software, 33(9):1-25, 2010. ISSN 1548-7660. URL http://www. jstatsoft. org/v33/i09.

C. Starr and R. Taggart. Biology: the Unity and Diversity of Life. Wadsworth Publishing Co Inc, Belmont, California, 5 edition, 1989 .

P. Stavem, A. Brgger, F. Devik, J. Flatby, C. B. van der Hagen, T. Henriksen, P. S. Hoel, H. Hst, K. Kett, and B. Petersen. Lethal acute gamma radiation accident at Kjeller, Norway: Report of a case. Acta Oncologica, 24(1):61-63, 1985. ISSN 0349-652X. URL http://www.ncbi.nlm.nih.gov/pubmed/2984904.

L. W. Sullivan, W. H. Adams, and Y. K. Liu. Induction of thrombocytopenia by thrombopheresis in man: Patterns of recovery in normal subjects during ethanol ingestion and abstinence. Blood, 49(2):197-207, 02 1977. ISSN 0006-4971. URL http://www.ncbi.nlm.nih.gov/pubmed/831873.

UNSCEAR. Sources, effects, and risks of ionizing radiation. 1988 report to the General Assembly. United Nations Scientific Committee on the Effects of Atomic Radiation, New York, 1988.

I. N. Usacheva and N. V. Raeva. Normal indices of the peripheral blood and bone marrow for the monkey macacus rhesus. Bulletin of Experimental Biology and Medicine, 54(5):1285-1287, 111963. ISSN 0007-4888. doi: 10.1007/BF00785609. URL http://link. springer.com/10.1007/BF00785609.

J. Valentin. Basic anatomical and physiological data for use in radiological protection: Reference values. ICRP Publication 89. Annals of ICRP, 32(3-4):1-277, 2002.

Y. Wang, H.-T. Tang, Z.-F. Xia, S.-H. Zhu, B. Ma, W. Wei, Y. Sun, and K.-Y. Lv. Factors affecting survival in adult patients with massive burns. Burns : journal of the International Society for Burn Injuries, 36(1):57-64, 02 2010. ISSN 1879-1409. doi: 10. 1016/j.burns.2009.04.014. URL http://www.ncbi.nlm.nih.gov/ pubmed/19487080.

V. Weisbach, H. Friedlein, A. Glaser, J. Zingsem, R. Zimmermann, and R. Eckstein. The influence of automated plateletpheresis on systemic levels of hematopoietic growth factors. Transfusion, 39(8):889-94, 08 1999. ISSN 0041-1132. URL http://onlinelibrary.wiley.com/doi/10.1046/j.15372995.1999.39080889.x/fullhttp://www.ncbi.nlm.nih.gov/ pubmed/10504126.

J. Wentz, D. Oldson, and D. Stricklin. Modeling the thrombopoietic effects of burn. Letters in Biomathematics, 1(1), 2014a.

J. Wentz, D. Oldson, and D. Stricklin. Mathematical models of human hematopoiesis following acute radiation exposure. Technical Report DTRA-TR-14-31, Defense Threat Reduction Agency, Fort Belvoir, VA, 2014b. URL http://oai.dtic.mil/oai/oai?verb= getRecord\&metadataPref $i \mathrm{x}=\mathrm{html} \&$ ident if $i$ er=ADA602115.

World Book Inc. Staff. Blood. In World Book Encyclopedia, page 407. Chicago: World Book, 1998.

S. P. Yarmonenko. Radiation sickness in man. In Radiobiology of Humans and Animals, pages 153-70. Mir Publishers, Moscow, 1988. URL http://www.getcited.org/pub/102915198.

M. R. Yeaman. Platelets in defense against bacterial pathogens. Cellular and molecular life sciences: CMLS, 67(4):525-44, 
02 2010. ISSN 1420-9071. doi: 10.1007/s00018-009-0210-4. URL http://www . pubmedcentral.nih.gov/articlerender.fcgi? artid=2809947\&tool=pmcentrez\&rendertype=abstract.

\section{Appendix A}

\section{A.1 Equations for mathematical model}

We assume that the thrombopoietic system is at a steady-state before radiation exposure: for $t<0, x_{1}^{u d}(t)=$ $\bar{x}_{1}^{u d}, x_{2}^{u d}(t)=\bar{x}_{2}^{u d}$, and $x_{3}(t)=\bar{x}_{3}$, where $\bar{x}_{1}^{u d}, \bar{x}_{2}^{u d}$, and $\bar{x}_{3}$ are the steady-state cell concentrations in the $X_{1}, X_{2}$, and $X_{3}$ compartments, respectively. Following acute exposure, we assume that radiosensitive cells in the $X_{1}$ and $X_{2}$ compartments instantaneously transition at time $t=0$ into their respective "damaged" compartments:

$$
\begin{aligned}
& x_{1}^{u d}(0)=\bar{x}_{1}^{u d}\left(1-\left(1-e^{-D / D_{1}^{0}}\right)^{n_{1}}\right) \\
& x_{2 I, i}^{u d}(0)=\frac{\bar{x}_{2}^{u d}}{n}\left(1-\left(1-e^{-D / D_{2}^{0}}\right)^{n_{2}}\right) \quad(i=1,2, \ldots, n / 2) \\
& x_{2 M, i}^{u d}(0)=\frac{\bar{x}_{2}^{u d}}{n}\left(1-\left(1-e^{-D / D_{2}^{0}}\right)^{n_{2}}\right) \quad(i=1,2, \ldots, n / 2) \\
& x_{3, i}(0)=\frac{\bar{x}_{3}}{m} \quad(i=1,2, \ldots, m) \\
& x_{1}^{d}(0)=\bar{x}_{1}^{u d}\left(1-e^{-D / D_{1}^{0}}\right)^{n_{1}} \\
& x_{2}^{d}(0)=\bar{x}_{2}^{u d}\left(1-e^{-D / D_{2}^{0}}\right)^{n_{2}}
\end{aligned}
$$

where $D$ is the radiation dose. The cell concentration values given in Eq. A.1-A.6 are then used as initial conditions for the following differential equation that describes the dynamics of mitotic progenitor repopulation, megakaryocyte maturation, and platelet decay (where we have used dot notation to represent time derivatives):

$$
\begin{aligned}
\dot{x}_{1}^{u d} & =B x_{1}^{u d}-\gamma x_{1}^{u d} \\
\dot{x}_{2 I, 1}^{u d} & =\gamma x_{1}^{u d}-n \delta x_{2 I, 1}^{u d} \\
\dot{x}_{2 I, i}^{u d} & =n \delta x_{2 I, i-1}^{u d}-n \delta x_{2 I, i}^{u d} \quad(i=2,3, \ldots, n / 2) \\
\dot{x}_{2 M, 1}^{u d} & =n \delta x_{2 I, n / 2}^{u d}-n \delta_{0} x_{2 M, 1}^{u d} \\
\dot{x}_{2 M, i}^{u d} & =n \delta_{0} x_{2 M, i-1}^{u d}-n \delta_{0} x_{2 M, i}^{u d} \quad(i=2,3, \ldots, n / 2) \\
\dot{x}_{3,1} & =\sigma n \delta_{0} x_{2 M, n / 2}^{u d}-m \psi x_{3,1} \\
\dot{x}_{3, i} & =m \psi x_{3, i-1}-m \psi x_{3, i} \quad(i=2,3, \ldots, m) \\
\dot{x}_{i}^{d} & =-\mu x_{i}^{d} \quad(i=1,2)
\end{aligned}
$$

where 


$$
\begin{aligned}
B & =\frac{\alpha}{1+\beta\left(\theta_{1}\left(x_{1}^{u d}+x_{1}^{d}\right)+\theta_{2}\left(x_{2}^{u d}+x_{2}^{d}\right)+\theta_{3} x_{3}\right)} \\
x_{2}^{u d} & =\sum_{i=1}^{n / 2} x_{2 I, i}^{u d}+x_{2 M, i}^{u d} \\
x_{3} & =\sum_{i=1}^{m} x_{3, i} \\
\delta & =\frac{1}{2 \tau_{2 I}} \\
\tau_{2 I} & =\left(\frac{1}{\tau_{2 I}^{\max }}+\left(\frac{1}{\tau_{2 I}^{\min }}-\frac{1}{\tau_{2 I}^{\max }}\right)\left(\frac{\frac{1}{\tau_{0} / 2}-\frac{1}{\tau_{2 I}^{\max }}}{\frac{1}{\tau_{2 I}^{\min }}-\frac{1}{\tau_{2 I}^{\max }}}\right)^{\left(\frac{x_{3}}{\bar{x}_{3}}\right)^{\lambda}}\right)
\end{aligned}
$$

where $\tau_{0}=1 / \delta_{0}, \tau_{2 I}^{\min }=\tau_{\min }-\tau_{0} / 2$, and $\tau_{2 I}^{\max }=\tau_{\max }-$ $\tau_{0} / 2$. Note that if $\tau_{\max }=\tau_{\min }=\tau_{0}$, the subcompartment model given by Eq. A.8-A.13, A.16, A.17 can be viewed as the result of applying the Linear Chain Trick (MacDonald, 1978) to the distributed delay model

$$
\begin{aligned}
\dot{x}_{2 I}^{u d} & =\gamma x_{1}^{u d}-F \\
\dot{x}_{2 M}^{u d} & =F-J \\
\dot{x}_{3} & =\sigma J-K \\
x_{2}^{u d} & =x_{2 I}^{u d}+x_{2 M}^{u d}
\end{aligned}
$$

where

$$
\begin{aligned}
F(t) & =\int_{-\infty}^{t} \gamma x_{1}^{u d}(\tau) g\left(t-\tau ; n / 2, n \delta_{0}\right) d \tau \\
J(t) & =\int_{-\infty}^{t} F(\tau) g\left(t-\tau ; n / 2, n \delta_{0}\right) d \tau \\
K(t) & =\int_{-\infty}^{t} \sigma J(\tau) g(t-\tau ; m, m \psi) d \tau
\end{aligned}
$$

and $g(x ; a, b)$ is the density of the gamma distribution with expectation $a / b$ and variance $\frac{a}{b^{2}}$. However, if $\tau_{\max } \neq \tau_{0}$ or $\tau_{\min } \neq \tau_{0}$ and the system is not in a steady-state, then $\delta$ is time dependent, in which case we cannot use the Linear Chain Trick to derive the subcompartment model given above. Nevertheless, Eq. A.20-A.22 provide motivation for Eq. A.8-A.13.

\section{A.2 Model predictions for relative compartment sizes}

When the system is at healthy, steady-state conditions, $x_{1}^{u d}=\bar{x}_{1}^{u d}, x_{2 I, i}^{u d}=\bar{x}_{2 I, i}^{u d}, i=1,2, \ldots, n / 2, x_{2 M, i}^{u d}=\bar{x}_{2 M, i}^{u d}$, $i=1,2, \ldots, n / 2, x_{3, i}=\bar{x}_{3, i}, i=1,2, \ldots, m$, and Eq. 8-13 of Appendix A.1 imply

$$
\begin{aligned}
& 0=\gamma \bar{x}_{1}^{u d}-n \delta \bar{x}_{2 I, 1}^{u d} \\
& 0=n \delta \bar{x}_{2 I, i-1}^{u d}-n \delta \bar{x}_{2 I, i}^{u d} \quad(i=2,3, \ldots, n / 2) \\
& 0=n \delta \bar{x}_{2 I, n / 2}^{u d}-n \delta_{0} \bar{x}_{2 M, 1}^{u d} \\
& 0=n \delta_{0} \bar{x}_{2 M, i-1}^{u d}-n \delta_{0} \bar{x}_{2 M, i}^{u d} \quad(i=2,3, \ldots, n / 2) \\
& 0=\sigma n \delta_{0} \bar{x}_{2 M, n / 2}^{u d}-m \psi \bar{x}_{3,1} \\
& 0=m \psi \bar{x}_{3, i-1}-m \psi \bar{x}_{3, i} \quad(i=2,3, \ldots, m)
\end{aligned}
$$

Moreover, under steady-state conditions $\delta=\delta_{0}$, so we -1 must have

$$
\begin{gathered}
\bar{x}_{2 I, 1}^{u d}=\bar{x}_{2 I, 2}^{u d}=\ldots=\bar{x}_{2 I, n / 2}^{u d}=\bar{x}_{2 M, 1}^{u d}=\bar{x}_{2 M, 2}^{u d}=\ldots=\bar{x}_{2 M, n / 2}^{u d} \\
\bar{x}_{3,1}=\bar{x}_{3,2}=\ldots=\bar{x}_{3, m}
\end{gathered}
$$

and

$$
\begin{aligned}
\frac{\bar{x}_{2 I, 1}^{u d}}{\bar{x}_{1}^{u d}} & =\frac{\gamma}{n \delta_{0}} \\
\frac{\bar{x}_{3,1}}{\bar{x}_{2 M, n / 2}^{u d}} & =\frac{\sigma n \delta_{0}}{m \psi}
\end{aligned}
$$

Equations A.16 and A.17 thus imply that, under steadystate conditions, $\bar{x}_{2}^{u d}=n \bar{x}_{2 I, 1}^{u d}=n \bar{x}_{2 M, n / 2}^{u d}$ and $\bar{x}_{3}=$ $m \bar{x}_{3,1}$, so under steady-state conditions the relative compartment sizes in humans are

$$
\begin{aligned}
& \frac{\bar{x}_{2}^{u d}}{\bar{x}_{1}^{u d}}=\frac{\gamma}{\delta_{0}}=\frac{0.26}{0.06}=4.3 \\
& \frac{\bar{x}_{3}}{\bar{x}_{2}^{u d}}=\frac{\sigma \delta_{0}}{\psi}=\frac{3000 \cdot 0.06}{0.11}=1636 \\
& \frac{\bar{x}_{3}}{\bar{x}_{1}^{u d}}=\frac{\sigma \gamma}{\psi}=\frac{3000 \cdot 0.26}{0.11}=7091
\end{aligned}
$$

and the relative compartment sizes in rhesus are

$$
\begin{aligned}
& \frac{\bar{x}_{2}^{u d}}{\bar{x}_{1}^{u d}}=\frac{\gamma}{\delta_{0}}=\frac{0.06}{0.26}=0.23 \\
& \frac{\bar{x}_{3}}{\bar{x}_{2}^{u d}}=\frac{\sigma \delta_{0}}{\psi}=\frac{3000 \cdot 0.26}{0.18}=4333 \\
& \frac{\bar{x}_{3}}{\bar{x}_{1}^{u d}}=\frac{\sigma \gamma}{\psi}=\frac{3000 \cdot 0.06}{0.18}=1000
\end{aligned}
$$




\section{Appendix B}

\section{B.1 Optimization Data and Model Predictions}

Table B4 and B5 provide a summary of the data used to optimize the thrombopoiesis model in rhesus and humans, respectively. Figure B1 provides comparisons of the rhesus optimization data with the model predictions (a comparison of the model with the 7 Gy optimization data is shown in Figure 3). Comparisons of the human optimization data with model predictions is given in a publicly available technical report (Wentz et al., 2014b). 
Table B4: Optimization data summary for rhesus thrombopoiesis model

\begin{tabular}{lllll}
\hline Study/Ref. & Dose-rate (cGy/min) & Dose (Gy) & Number & Baseline $\left(10^{3} \mu L^{-1}\right)$ \\
\hline Data obtained from Neumedicines & 60 & 5.7 & 15 & 456.34 \\
Data obtained from Neumedicines & 60 & 6.0 & 12 & 454.63 \\
Data obtained from Neumedicines & 60 & 6.3 & 15 & 496.87 \\
Data obtained from Neumedicines & 60 & 6.6 & 15 & 509.90 \\
Basile et al. (2012) & 60 & 6.7 & 8 & 456.00 \\
Data obtained from Neumedicines & 60 & 6.9 & 16 & 474.85 \\
Gluzman-Poltorak et al. (2014a); Data & 60 & 7.0 & 32 & 416.92 \\
obtained from Neumedicines & & & \\
Data obtained from Neumedicines & 60 & 7.2 & 7.4 & 467.23 \\
Data obtained from Neumedicine & 60 & 4.0 & $30^{*}$ & 431.50 \\
Authier et al. (2013) & $43.6-57.9$ & 6.0 & $30^{*}$ & 411.73 \\
Authier et al. (2013) & $43.6-57.9$ & 6.34 & $30^{*}$ & 418.06 \\
Authier et al. (2013) & $43.6-57.9$ & 12.1 & $30^{*}$ & 396.81 \\
Authier et al. (2013) & 94.8 & & \\
\hline
\end{tabular}

All rhesus monkeys were irradiated using a Cobalt-60 source.

* The number of animals per group is assumed to be 30 based on a total of 120 animals for the entire study as reported in the reference.

Table B5: Optimization data summary for human thrombopoiesis model

\begin{tabular}{llcc}
\hline Incident/Subject & Ref. & Dose (Gy) & Baseline $\left(10^{3} \mu L^{-1}\right)$ \\
\hline Los Alamos 1945-2 & Hempelmann et al. (1952) & 0.12 & $819.26^{a}$ \\
UT CARL 1971 & Andrews et al. (1961)* & 2.6 & $350.63^{a}$ \\
Argonne 1952-I & Bond et al. (1965) & 1.5 & $111.23^{a}$ \\
Y-12 1958-B & Andrews et al. (1961)* & 2.97 & $161.25^{a}$ \\
Y-12 1958-C & Andrews et al. (1961)* & 3.73 & $181.25^{a}$ \\
Y-12 1958-A & Bond et al. (1965) & $289.56^{a}$ \\
Vinca 1958-B & Jammet et al. (1959) & 4.02 & $210.31^{a}$ \\
Vinca 1958-H & Jammet et al. (1959) & 4.51 & $233.73^{a}$ \\
Vinca 1958-M & Jammet et al. (1959) & 5.74 & $219.88^{a}$ \\
Vinca 1958-G & Jammet et al. (1959) & 5.35 & $250.00^{b}$ \\
Vinca 1958-D & Jammet et al. (1959) & 5.40 & $250.00^{b}$ \\
Vinca 1958-V & Jammet et al. (1959) & 6.11 & $221.29^{a}$ \\
Yarmonenko 1988-Z & Yarmonenko (1988) & 9.8 & $140.21^{a}$ \\
Nesvizh 1991 & Baranov et al. (1994) & 12.5 & $106.75^{a}$ \\
Cancer patients 1958-mean of $18^{c}$ & Miller et al. (1958) & 1.0 & $197.25^{d}$ \\
Cancer patients 1958-mean of $12^{c}$ & Miller et al. (1958) & 1.5 & $234.25^{d}$ \\
Cancer patients 1958-mean of 30 & Miller et al. (1958) & 2.0 & $155.75^{d}$ \\
\hline
\end{tabular}

Radiation exposure studies used for optimization of the human thrombopoiesis model, including the reference for the data (Ref.) the approximate radiation dose, and the estimated baseline peripheral thrombocyte concentration.

${ }^{a}$ Mean of up to four data points collected within 3 days of radiation exposure

${ }^{b}$ Mean platelet concentration observed in humans Valentin (2002)

${ }^{c}$ Subjects had non hematopoietic cancer and there was no mention of any pre-treatment in the source documentation.

${ }^{d}$ Mean of pre-radiation data points

* Data provided by Ron Goans 

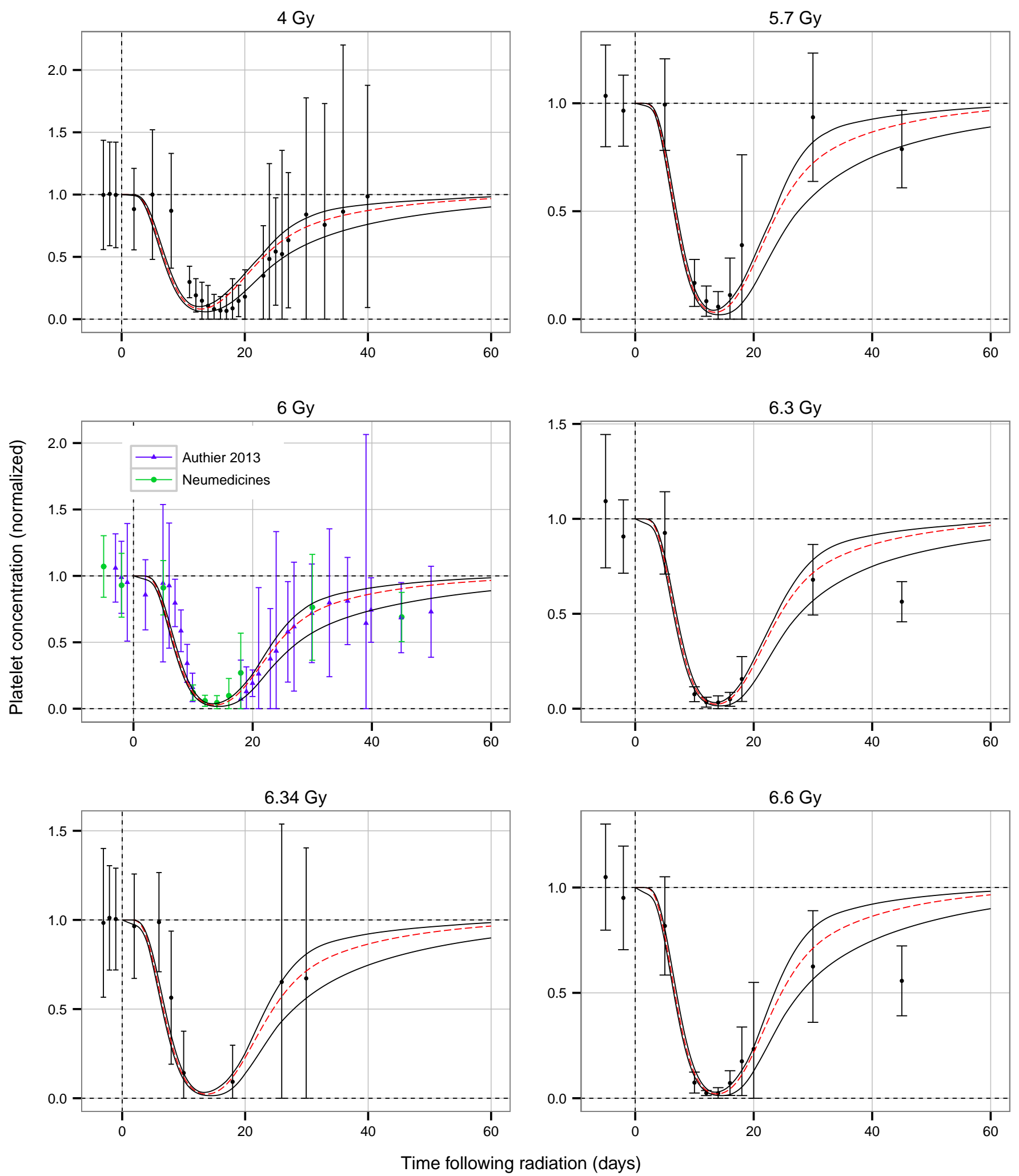

Figure B1: Thrombopoiesis model overlaid on rhesus optimization data not shown in paper. Optimized simulation (red dashed line) shown along with range (black lines) based on sampling of parameter values from MCMC analysis. 
6.7 Gy

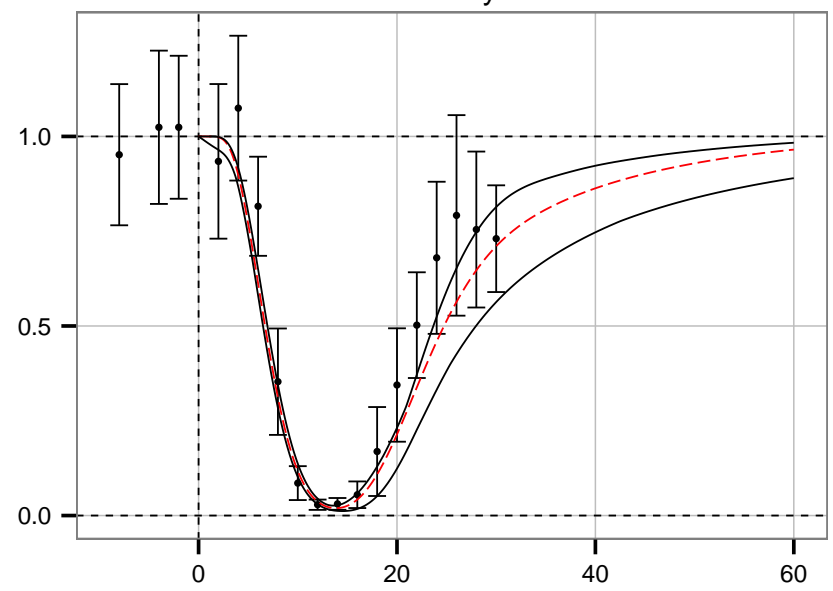

7.2 Gy

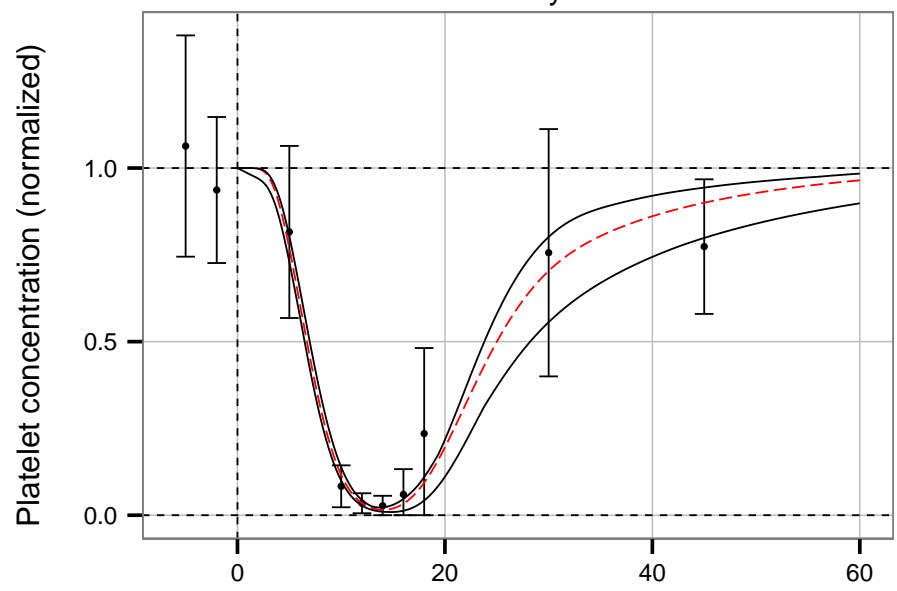

12.1 Gy

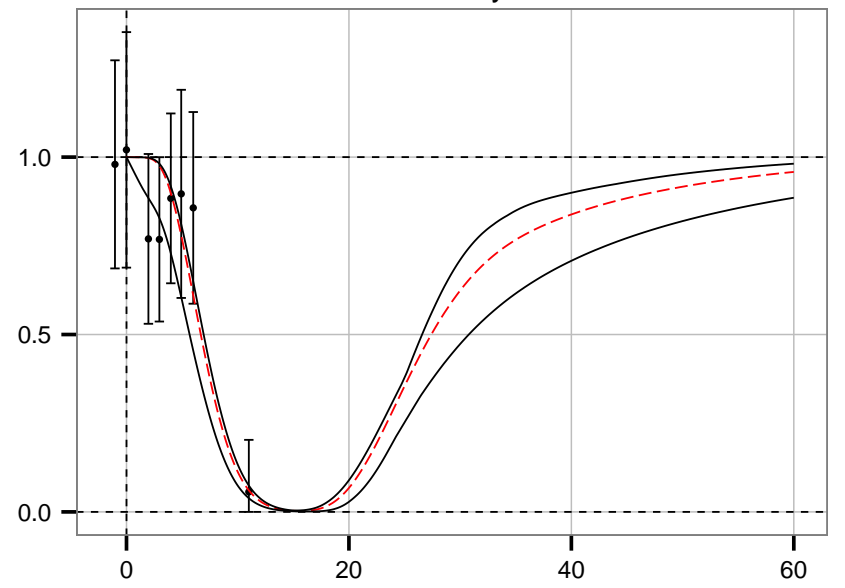

$6.9 \mathrm{~Gy}$

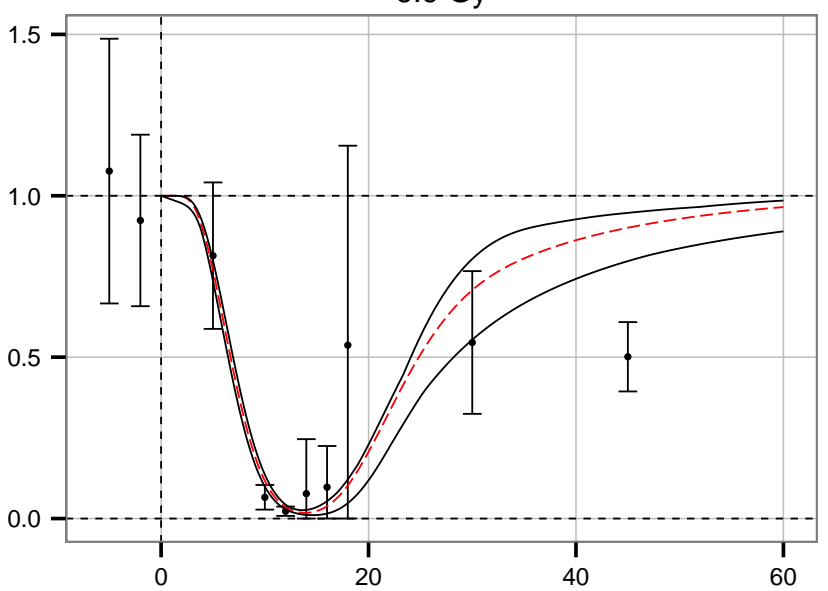

$7.4 \mathrm{~Gy}$

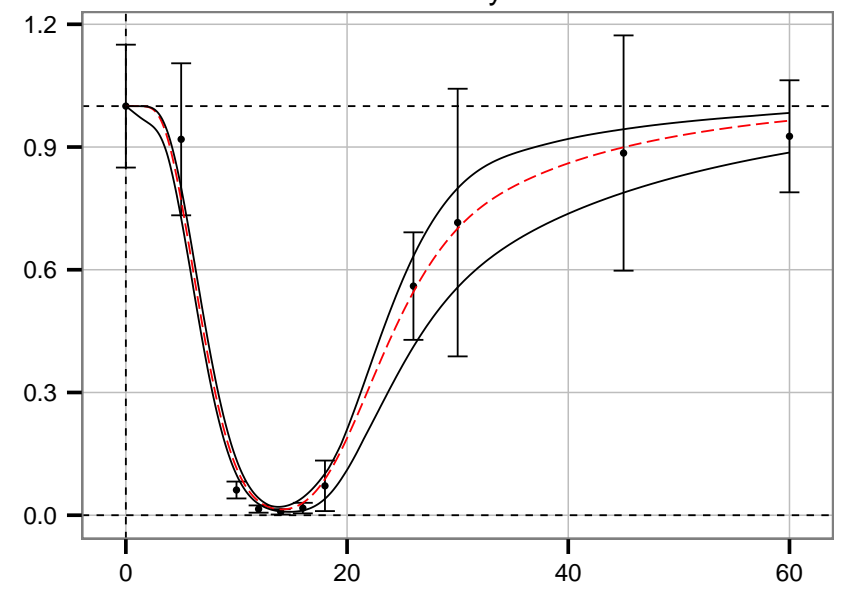

Time following radiation (days)

Figure B1: (cont.) Thrombopoiesis model overlaid on rhesus optimization data not shown in paper. Optimized simulation (red dashed line) shown along with range (black lines) based on sampling of parameter values from MCMC analysis. 